\title{
COSMOLOGICAL IMPACT OF POPULATION III BINARIES
}

\author{
Ke-Jung Chen $^{1,2}$, Volker Bromm ${ }^{3}$, Alexander Heger ${ }^{2,4,5}$, Myoungwon Jeon ${ }^{3}$, And Stan Woosley ${ }^{1}$ \\ ${ }^{1}$ Department of Astronomy \& Astrophysics, University of California, Santa Cruz, CA 95064, USA; kchen@ucolick.org \\ ${ }^{2}$ School of Physics and Astronomy, University of Minnesota, Minneapolis, MN 55455, USA \\ ${ }^{3}$ Department of Astronomy, University of Texas, Austin, TX 78712, USA \\ ${ }^{4}$ Monash Centre for Astrophysics, School of Mathematical Sciences, Monash University, Victoria 3800, Australia \\ 5 Joint Institute for Nuclear Astrophysics, University of Notre Dame Notre Dame, IN 46556, USA \\ Received 2014 July 25; accepted 2015 January 14; published 2015 March 16
}

\begin{abstract}
We present the results of the stellar feedback from Population III (Pop III) binaries by employing improved, more realistic Pop III evolutionary stellar models. To facilitate a meaningful comparison, we consider a fixed mass of $60 M_{\odot}$ incorporated in Pop III stars, either contained in a single star, or split up in binary stars of $30 M_{\odot}$ each or an asymmetric case of one 45 and one $15 M_{\odot}$ star. Whereas the sizes of the resulting $\mathrm{H}$ in regions are comparable across all cases, the He III regions around binary stars are significantly smaller than that of the single star. Consequently, the $\mathrm{He}^{+} 1640 \AA$ recombination line is expected to become much weaker. Supernova (SN) feedback exhibits great variety due to the uncertainty in possible explosion pathways. If at least one of the component stars dies as a hypernova about 10 times more energetic than conventional core-collapse $\mathrm{SNe}$, the gas inside the host minihalo is effectively blown out, chemically enriching the intergalactic medium (IGM) to an average metallicity of $10^{-4}-10^{-3} Z_{\odot}$, out to $\sim 2 \mathrm{kpc}$. The single star, however, is more likely to collapse into a black hole, accompanied by at most very weak explosions. The effectiveness of early chemical enrichment would thus be significantly reduced, in contrast to the lower mass binary stars, where at least one component is likely to contribute to heavy element production and dispersal. Important new feedback physics is also introduced if close binaries can form high-mass X-ray binaries, leading to the pre-heating and -ionization of the IGM beyond the extent of the stellar $\mathrm{H}$ II regions.
\end{abstract}

Key words: early universe - galaxies: formation - radiative transfer - stars: formation - stars: Population III supernovae: general

\section{INTRODUCTION}

One of the paramount problems in modern cosmology is to elucidate how the first generation of luminous objects, stars, accreting black holes (BHs), and galaxies shaped the early universe at the end of the cosmic dark ages (Barkana \& Loeb 2001; Loeb \& Furlanetto 2012; Wiklind et al. 2013). A key driver of this grand cosmic transformation was the gradual enrichment of the pristine universe with heavy chemical elements in the wake of the first supernova (SN) explosions (reviewed in Karlsson et al. 2013). According to the modern theory of cosmological structure formation (e.g., Mo et al. 2010), the hierarchical assembly of dark matter (DM) halos provided the gravitational potential wells that allowed gas to form stars and galaxies inside them. Extending this model to the highest redshifts, one can determine the sites where the first stars, the so-called Population III (Pop III) stars, formed out of the pure $\mathrm{H} / \mathrm{He}$ gas created in the big bang. Within this framework, Pop III stars are predicted to form inside DM minihalos with total masses (DM and gas) of about $10^{6} M_{\odot}$ at redshifts of $z \sim 20-30$ (Couchman \& Rees 1986; Haiman et al. 1996; Tegmark et al. 1997; Bromm \& Larson 2004; O'Shea et al. 2008; Bromm et al. 2009).

The first stars affected the early universe in several different ways. Massive Pop III stars were strong emitters of hydrogen and helium ionizing photons that built up extensive $\mathrm{H}_{\text {II, }} \mathrm{He}$ II, and He III regions (Yoshida et al 2007). The metals forged in Pop III stars later were dispersed into the intergalactic medium (IGM) when they died as $\mathrm{SNe}$, thus quickly polluting the primordial gas such that the second generation of (Population II) stars could emerge. It is convenient to classify
Pop III feedback mechanisms into different classes (Ciardi \& Ferrara 2005), specifically radiative feedback (O'Shea et al. 2005; Abel et al. 2007; Susa 2007; Yoshida et al 2007; Whalen et al. 2008, 2010; Greif et al. 2009a; Hasegawa et al. 2009), mechanical and chemical feedback due to SNe (Wise \& Abel 2008; Greif et al. 2010; Ritter et al. 2012), and X-ray feedback from accreting BH remnants (Kuhlen \& Madau 2005; Alvarez et al. 2009; Jeon et al. 2012, 2014; Xu et al. 2014).

In the Pop III star-forming regions, metal cooling was absent because the primordial gas consisted almost exclusively of hydrogen ( $\sim 76 \%$ by mass) and helium $(\sim 24 \%)$. Molecular hydrogen was thus the dominant coolant, but owing to its quantum-mechanical structure, it was unable to cool the gas to the low temperatures typically encountered in star-forming clouds today. The primordial gas, therefore, remained relatively warm, with typical temperatures of several hundred kelvin. Hence, the Jeans mass was correspondingly larger as well, leading to the expectation that Pop III stars might have been more massive than stars formed today, with a predicted mass scale of 50-100 $M_{\odot}$ (Bromm et al. 1999, 2002; Nakamura \& Umemura 2001; Abel et al. 2002; Omukai \& Palla 2003). Because of their high surface temperatures (Bond et al. 1984; Bromm et al. 2001b; Schaerer 2002), Pop III stars could effectively produce copious amounts of ionizing UV photons. Extended $\mathrm{H}_{\text {II }}$ regions with size of several kiloparsecs were created before the stars died. Given the shallowness of the gravitational potential well of the host DM halos, the surrounding gas was subject to strong photo-heating, thus being able to easily escape the host minihalos (Whalen et al. 2004; Alvarez et al. 2006). This photo-evaporation suppressed further star formation inside the minihalos, thus 
delaying further star formation until more massive host halos emerged (Bromm \& Yoshida 2011).

The character of Pop III feedback sensitively depends on the fate encountered by massive Pop III stars when they die after their short lifetime of a few million years to trigger an $\mathrm{SN}$ explosion, or directly collapse into BHs. Those Pop III stars with masses of 140-260 $M_{\odot}$ are thought to die as pairinstability supernovae (PSNe; Barkat et al. 1967; Heger \& Woosley 2002; Chen et al. 2014), although this mass range may have to be revised in the case of rapidly rotating progenitors (Chatzopoulos \& Wheeler 2012). Unlike gravitationally powered core-collapse supernovae (CCSNe), PSNe are hyper-energetic thermonuclear explosions, not leaving any compact remnant behind. Because vast amounts of metals are ejected during a PSN explosion, even a single event could enrich about $10^{7} M_{\odot}$ of primordial gas up to $10^{-4}-10^{-3} Z_{\odot}$ (Karlsson et al. 2008; Wise \& Abel 2008; Sakuma \& Susa 2009; Greif et al. 2010). Even such a trace amount of metals could change the subsequent star formation process and might cause a transition of the stellar initial mass function (IMF) from the top-heavy mode predicted for Pop III stars to the standard IMF for later (Pop I and Pop II) generations with typical masses comparable to that of our Sun (Bromm et al. 2001a; Omukai et al. 2005; Maio et al. 2010; Wise et al. 2012).

Results from stellar archaeology (Beers \& Christlieb 2005; Frebel et al. 2005), which studies the most metal-poor stars in the Local Group that retained the imprints from nucleosynthesis in the early universe, possibly including those from Pop III stars, in general do not support the chemical abundance pattern predicted for PSN enrichment (Tumlinson 2006). Theoretical PSN yields exhibit a pronounced odd-even effect, resulting from a low neutron excess (Heger \& Woosley 2002). In addition, the lack of any neutron capture process results in the absence of all elements heavier than the Fe peak (no r- or sprocess). The Pop III CCSN models (Umeda \& Nomoto 2003; Heger \& Woosley 2010), however, can produce abundance patterns in good accord with the observation of metal-poor stars. Recent simulations of Pop III star formation that take into account the radiation-hydrodynamical feedback from the growing central protostar have shown that accretion can be halted, thus preventing the formation of stars more massive than $50 M_{\odot}$ (Hosokawa et al. 2011; Stacy et al. 2012; Hirano et al. 2014). That implies that Pop III stars typically might die in a CCSN, instead of a PSN, in agreement with the observations. Furthermore, recent cosmological simulations with extremely high resolution have shown that the primordial gas cloud is able to fragment and produce stars of relatively lower mass of tens of solar masses (Turk et al. 2009; Stacy et al. 2010; Greif et al. 2011), organized in binaries or multiple stellar systems. These simulations suggest that binary systems may be the typical channel for primordial star formation in minihalos (Stacy \& Bromm 2013). Since these simulations only follow the protostellar assembly process for at most $\sim 10^{3}$ $\mathrm{yr}$, it is not yet clear how the final mass spectrum will look like (Bromm 2013). One key uncertainty is the degree of merging of neighboring protostars (Greif et al. 2012). However, it appears likely that the first stars in minihalos typically formed in binary or small multiple systems. Since the evolution of binary systems and their final fate are very different from those of single stars, it is worthwhile to investigate whether or not binary Pop III stars lead to significantly altered feedback effects.

Since their evolution is quite different from a single star, it is worth investigating how the Pop III binary systems affected the IGM and their host halos that later merged into the first galaxies (Ricotti et al. 2002; Wise \& Abel 2008; Johnson et al. 2009, 2013; Wise \& Cen 2009; Xu et al. 2013; Wise et al. 2014). The evolving binaries might exert different feedback mechanisms, through the emission of UV and X-ray ionizing photons and SN explosions, all of which may be quite different from the feedback of the single stars, which has been well documented in the literature. Therefore, we first study the impact of the first massive stars of masses $60,45,30$, and $15 M_{\odot}$ on their parent halos.

We study the possible impacts of the first binary systems on the IGM and present the results of cosmological simulations by considering possible outcomes of the Pop III binary models with stars of $45 M_{\odot}+15 M_{\odot}(\mathrm{S} 45+\mathrm{S} 15)$ and $30 M_{\odot}+30 M_{\odot}$ (S30+S30). Our binary models consider the non-interacting binaries during their stellar evolution. However, more realistic binary models might have a much wider range of outcomes (Langer 2012).

The structure of this paper is as follows: In Section 2, we describe our initial setup, as well as our numerical methods. A discussion of our protostellar evolution models, both for single and binary stars, follows in Section 3. The simulation results are presented in Section 4, and we conclude by discussing the broader implications in Section 5. All of the results presented in this paper use physical coordinates instead of comoving coordinates.

\section{NUMERICAL METHODOLOGY}

\subsection{Problem Setup}

The primary code used for our simulations is the well-tested, massively parallel cosmological code GADGET (Springel 2005), which computes gravitational forces with a hierarchical tree algorithm and represents fluids by means of smoothed particle hydrodynamics (SPH). In order to simulate the feedback exerted by the first stars, additional physical processes, such as cooling and chemistry of the primordial gas, radiative transfer of ionizing photons, and SN explosions, are required and have been implemented in GADGET.

Our simulations employ the same initial conditions as in Greif et al. (2010), starting at $z=100$ in a periodic box of linear size of $1 \mathrm{Mpc}$ (comoving). We choose $\Lambda \mathrm{CDM}$ cosmological parameters with matter density $\Omega_{m}=0.3$, baryon density $\Omega_{b}=0.04$, Hubble constant $H_{0}=70 \mathrm{~km} \mathrm{~s}^{-1} \mathrm{Mpc}^{-1}$, spectral index $n_{s}=1.0$, and normalization $\sigma_{8}=0.9$, based on the WMAP cosmic microwave background measurement (Komatsu et al. 2009). Greif et al. (2010) used a standard hierarchical zoom-in technique, generating the highest mass resolution covering the Lagrangian region where the first galaxy is destined to form. That way, all the relevant finestructure, specifically the minihalo progenitors of the first galaxy, can be resolved. The resulting mass of the DM and gas particles in the highest resolution region is $m_{\mathrm{DM}} \sim 33 M_{\odot}$ and $m_{\mathrm{SPH}} \sim 5 M_{\odot}$, respectively. Because the molecular hydrogen cooling in primordial gas imprints a characteristic density of $n_{\mathrm{H}}=10^{4} \mathrm{~cm}^{-3}$ and temperature of $200 \mathrm{~K}$ (Bromm et al. 2002), our simulations marginally resolve the corresponding Jeans mass of $M_{J} \simeq 500 M_{\odot}$. Our cooling module 
and chemistry network are based on Greif et al. (2010), and include all relevant cooling mechanisms of primordial gas, such as $\mathrm{H}$ and $\mathrm{He}$ collisional ionization, excitation and recombination cooling, bremsstrahlung, and inverse Compton cooling; in addition, the collisional excitation cooling via $\mathrm{H}_{2}$ and $\mathrm{HD}$ is also taken into account. For $\mathrm{H}_{2}$ cooling, collisions with protons and electrons are explicitly included. The chemical network includes $\mathrm{H}, \mathrm{H}^{+}, \mathrm{H}^{-}, \mathrm{H}_{2}, \mathrm{H}_{2}^{+}, \mathrm{He}, \mathrm{He}^{+}, \mathrm{He}^{++}$, and $\mathrm{e}^{-}, \mathrm{D}, \mathrm{D}^{+}$, and HD.

State-of-the-art cosmological simulations can potentially use billions of particles to model the formation of the universe. However, it is still extremely challenging to resolve mass scales from galaxies $\left(10^{10} M_{\odot}\right)$ to individual stars $\left(1 M_{\odot}\right)$. For example, the resolution length in our simulation is about 1 pc, many orders of magnitude removed from truly stellar scales. Hence, modeling the process of star formation on cosmological scales from first principles is currently still out of reach. A viable strategy to treat star formation and its feedback is to employ subgrid models, where sink particles approximately represent unresolved single stars, or clusters thereof. The sinks can then act as sources of radiation, with luminosities and spectra chosen in accordance with stellar structure and evolution models, and eventually as sites for SN explosions. Another reason to use sink particles is to overcome the socalled "Courant myopia". When the gas density somewhere inside the computational box becomes increasingly high, the SPH smoothing length decreases, in turn enforcing the adoption of smaller and smaller timesteps, according to the Courant condition. When encountering a runaway collapse, the simulation would effectively grind to a halt or fail. Creating sink particles allows us to bypass this numerical bottleneck, such that the simulations can be followed beyond the initial collapse, where much of the interesting physics, related to the stellar feedback, occurs. We here apply the sink particle algorithm of Johnson \& Bromm (2007). The key criterion for sink creation, and subsequent accretion of further SPH particles, is that the gas density exceeds a pre-specified density threshold, $n_{c} \sim 10^{4} \mathrm{~cm}^{-3}$, but we also test for gravitational boundedness, and whether the gas is part of a converging flow, where $\nabla \cdot \boldsymbol{v}<0$. The sink particles provide markers for the position of a Pop III star and its remnants, such as a $\mathrm{BH}$ or $\mathrm{SN}$, to which detailed subgrid physics can be supplied.

\subsection{Radiative Transfer}

When a Pop III star has formed inside the minihalo, the sink particle representing it immediately turns into a point source of ionizing photons to mimic the birth of a star. The rate of ionizing photons emitted depends on the physical size of the star and its surface temperature based on our stellar subgrid models. Instead of simply assuming constant rates of emission, we use the results of one-dimensional (1D) stellar evolution calculations from Heger \& Woosley (2010) to construct the luminosity history of Pop III stars. Indeed, luminosities exhibit considerable time variability when taking the evolution off the main sequence into account. The photons streaming from the star then establish an ionization front and build up $\mathrm{H}$ II regions. To trace the propagation of photons and the ionization front, we use the ray-tracing algorithm from (Greif et al. 2009a), which solves the ionization front equation in a spherical grid by tracking $10^{5}$ rays with 500 logarithmically spaced radial bins around the photon source. The radiation transport is coupled to
Table 1

Stellar Lifetimes and Fates

\begin{tabular}{lcccccc}
\hline \hline Model & $\begin{array}{c}\text { Mass } \\
\left(M_{\odot}\right)\end{array}$ & $\begin{array}{c}\text { MS } \\
(\mathrm{Myr})\end{array}$ & $\begin{array}{c}\text { Post-MS } \\
(\mathrm{Myr})\end{array}$ & $\begin{array}{c}\text { Total } \\
(\mathrm{Myr})\end{array}$ & Fates & $\begin{array}{c}\text { Metal Yield } \\
\left(M_{\odot}\right)\end{array}$ \\
\hline S15 & 15 & 9.478 & 1.031 & 10.51 & SN & 1.388 \\
S30 & 30 & 5.208 & 0.509 & 5.77 & BH, HN & 6.876 \\
S45 & 45 & 3.995 & 0.394 & 4.39 & BH, HN & 13.26 \\
S60 & 60 & 3.426 & 0.345 & 3.77 & BH, HN & 20.66 \\
\hline
\end{tabular}

Table 2

Summary of Assumed Stellar Fates

\begin{tabular}{lccll}
\hline \hline Type & $\begin{array}{c}\text { Masses } \\
\left(M_{\odot}\right)\end{array}$ & $\begin{array}{c}E_{\text {sn }} \\
(\mathrm{B})\end{array}$ & Mass Ejection & Notes \\
\hline SN & $\lesssim 25$ & 1.2 & all but $\sim 1.5 M_{\odot}$ & leaves neutron star \\
BH & $\gtrsim 25$ & 0 & None & complete collapse to BH \\
HN & $\gtrsim 25$ & 10 & $\sim 90 \%$ & energetic explosion \\
\hline
\end{tabular}

Note. $E_{\mathrm{sn}}$ is the explosion energy.

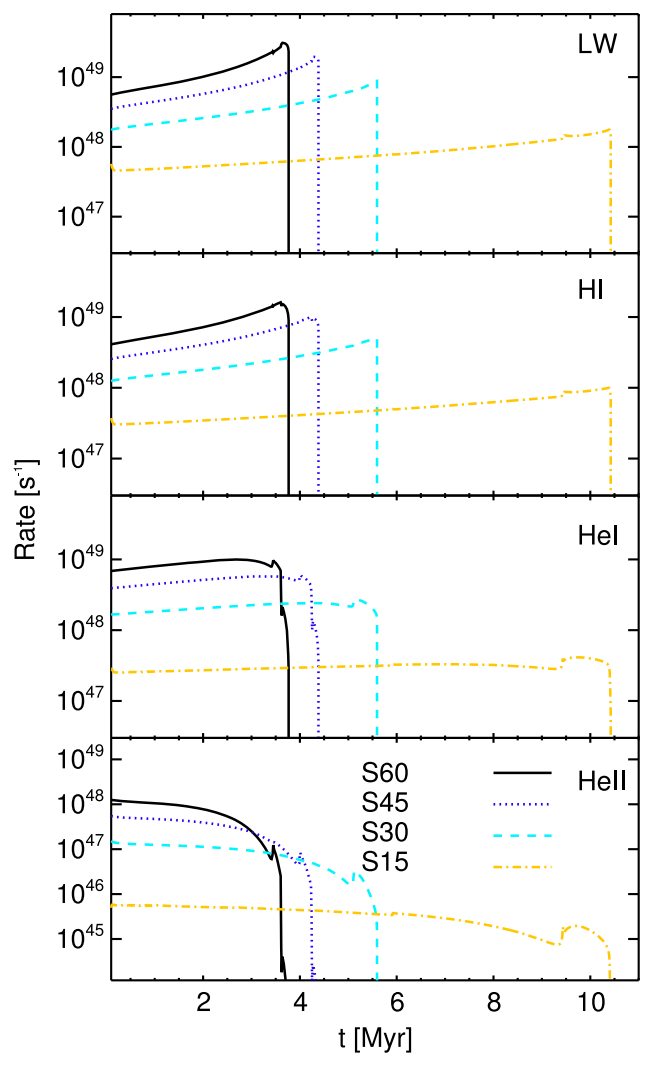

Figure 1. Evolution of UV flux of 60, 45, 30, and $15 M_{\odot}$ Pop III stars. During the main sequence stage, rates are close to constant. After leaving the main sequence, the luminosity of the stars increases due to the expansion of the envelope, leading a drop in temperature. This leads to an increase in the $\mathrm{H}_{\mathrm{I}}$ ionizing photon rate (lower energy band), but a decrease in the rate of He II ionizing photons (higher energy band).

the hydrodynamics of the gas through its chemical and thermal evolution. The transfer of the $\mathrm{H}_{2}$-dissociating photons in the Lyman-Werner $(\mathrm{LW})$ band $(11.2-13.6 \mathrm{eV})$ is also included.

For completeness, we here briefly present the key features of the ray-tracing algorithm, and refer the reader to Greif et al. (2009a) for details and tests. To begin with, particle positions are transformed from Cartesian to spherical coordinates, i.e., 
Table 3

Number of Ionizing Photons Emitted Over the Lifetime of a Star

\begin{tabular}{lcccc}
\hline \hline Model & Mass & $\begin{array}{c}\mathrm{H}_{\mathrm{I}} \\
\left(10^{63}\right)\end{array}$ & $\begin{array}{c}\mathrm{He}_{\mathrm{I}} \\
\left(10^{63}\right)\end{array}$ & $\begin{array}{c}\mathrm{He} \text { II } \\
\left(10^{61}\right)\end{array}$ \\
\hline S15 & 15 & 0.64 & 0.16 & 0.10 \\
S30 & 30 & 1.82 & 0.72 & 1.37 \\
S45 & 45 & 2.98 & 1.45 & 4.34 \\
S60 & 60 & 4.18 & 2.21 & 8.31 \\
\hline
\end{tabular}

Table 4

Summary of Binary Model Characteristics

\begin{tabular}{lccccc}
\hline \hline Case & $\begin{array}{c}\text { Masses } \\
\left(M_{\odot}\right)\end{array}$ & $\begin{array}{c}\text { Separation } \\
(\text { distance })\end{array}$ & $\begin{array}{c}\text { Fate } \\
1\end{array}$ & $\begin{array}{c}\text { Fate } \\
2\end{array}$ & $\begin{array}{c}\text { metals yields } \\
\left(M_{\odot}\right)\end{array}$ \\
\hline I & $30+30$ & wide & HN & HN & 13.74 \\
II & $30+30$ & wide & BH & BH & 0.00 \\
III & $45+15$ & close & BH & WD & 0.00 \\
\hline
\end{tabular}

Table 5

Number of Ionizing Photons Emitted Over the Lifetime of Binary Models

\begin{tabular}{lcccr}
\hline \hline Model & $\begin{array}{c}\mathrm{H}_{\mathrm{I}} \\
\left(10^{63}\right)\end{array}$ & $\begin{array}{c}\mathrm{He}_{\mathrm{I}} \\
\left(10^{63}\right)\end{array}$ & $\begin{array}{c}\text { He II } \\
\left(10^{61}\right)\end{array}$ & $\begin{array}{c}t_{*}{ }^{\mathrm{a}} \\
(\mathrm{Myr})\end{array}$ \\
\hline S30+S30 & 3.64 & 1.44 & 2.74 & 5.77 \\
S45+S15 & 3.62 & 1.61 & 4.43 & 10.51 \\
S60 & 4.18 & 2.21 & 8.31 & 3.77 \\
\hline
\end{tabular}

${ }^{\text {a }}$ Lifetime of a binary star (longest-lived component).

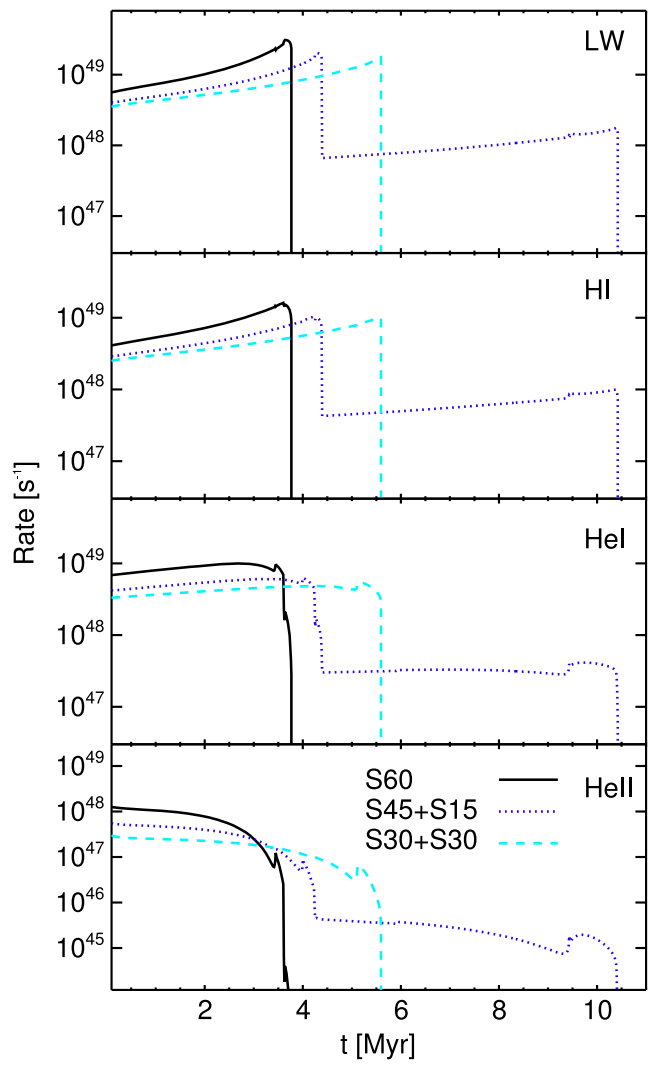

Figure 2. UV fluxes of the first binary stars. Note that fluxes are calculated by summing over the component stars. The resulting emission histories are quite distinct, with extended late-time flux when lower mass components are present.
Table 6

Summary of Feedback Models and Results

\begin{tabular}{cll}
\hline \hline Type of Star & Stellar Model and Feedback & Results (Figure: No) \\
\hline Single & S15 Rad & 3,5 \\
& S15 Rad+CCSN & 6,7 \\
S15 Rad+BH & 6 \\
S30 Rad & 3,5 \\
S30 Rad+HN & 6,7 \\
S30 Rad+BH & 6 \\
S45 Rad & 3,5 \\
S45 Rad+HN & $6,7,9$ \\
S45 Rad+BH & 6 \\
S60 Rad & $3,4,5$ \\
S60 Rad+HN & $6,7,8,9,11$ \\
Sinary & $8,9 \mathrm{Rad}+$ WSN & 6 \\
\hline S60 Rad+BH & 4,5 \\
\cline { 2 - 3 } & S45+S15 Rad & 9 \\
& S45+S15 Rad+HN & 10 \\
S45+S15 Rad+X-ray & 4,5 \\
& S30+S30 Rad & 8,9 \\
S30+S30 Rad+HN & 8,9 \\
\hline
\end{tabular}

Note. Rad presents the radiative feedback from the UV radiation. BH (black hole), CCSN (core-collapse SN), WSN (weak SN), and HN (hypernova) are different fates of the star.

radius $(r)$, zenith angle $(\theta)$, and azimuth angle $(\phi)$. The effective volume of each particle is $\sim h^{3}$, where $h$ is the SPH smoothing length. The corresponding sizes in spherical coordinates are $\Delta r=h, \Delta \theta=h / r$, and $\Delta \phi=h / r \sin \theta$. Using spherical coordinates facilitates the convenient calculation of the ionization front around the star,

$$
n_{n} r_{I}^{2} \frac{d r_{I}}{d t}=\frac{\dot{N}_{\text {ion }}}{4 \pi}-\alpha_{B} \int_{0}^{r_{I}} n_{e} n_{+} r^{2} d r
$$

where $r_{I}$ is the position of the ionization front, $\dot{N}_{\text {ion }}$ represents the number of ionizing photons emitted from the star per second, $\alpha_{B}$ is the case $B$ recombination coefficient, and $n_{n}, n_{e}$, and $n_{+}$are the number densities of neutral particles, electrons, and positively charged ions, respectively. The recombination coefficient is assumed to be constant at temperatures around $2 \times 10^{4} \mathrm{~K}$. The ionizing photon rates are

$$
\dot{N}_{\text {ion }}=\frac{\pi L_{*}}{\sigma_{\mathrm{SB}} T_{\mathrm{eff}}^{4}} \int_{\nu_{\min }}^{\infty} \frac{B_{\nu}}{h_{\mathrm{P}} \nu} d \nu
$$

where $h_{\mathrm{P}}$ is Planck's constant, $\sigma_{\mathrm{SB}}$ the Stefan-Boltzmann constant, $L_{*}$ the luminosity of the massive star, and $\nu_{\min }$ the ionization threshold for $\mathrm{HI}, \mathrm{He} \mathrm{I}$, and $\mathrm{He}$ II. By assuming a blackbody spectrum $B_{\nu}$ with effective temperature, $T_{\text {eff }}$, the flux of a Pop III star can be written:

$$
F_{\nu}=\frac{L_{*}}{4 \sigma_{\mathrm{SB}} T_{\mathrm{eff}}^{4} r^{2}} B_{\nu}
$$

The size of the $\mathrm{H}$ II region is determined by solving Equation 1 . The particles within the $\mathrm{H}$ II regions store information about their distance from the star, which is used to calculate the 

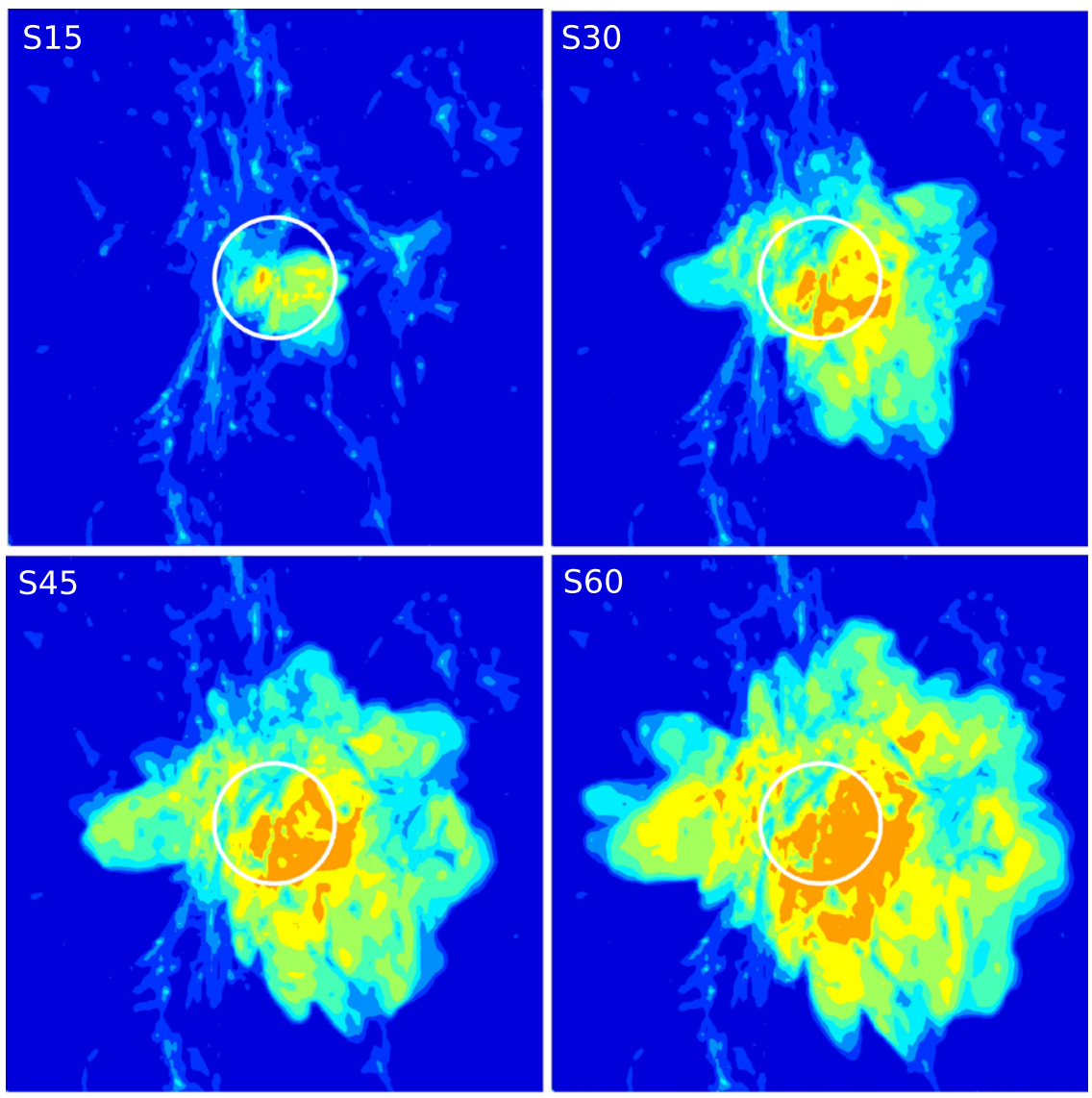

$\log (T / K)$

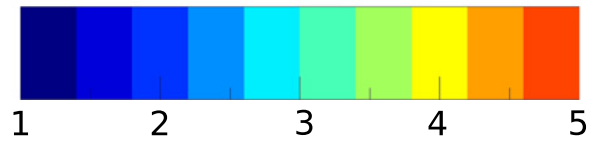

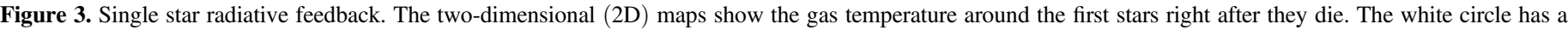
radius of $1 \mathrm{kpc}$, and its center is located at the position of the first star.

ionization and heating rates,

$$
\begin{aligned}
k_{\text {ion }} & =\int_{\nu_{\min }}^{\infty} \frac{F_{\nu} \sigma_{\nu}}{h_{\mathrm{P}} \nu} d \nu, \\
\Gamma & =n_{n} \int_{\nu_{\min }}^{\infty} F_{\nu} \sigma_{\nu}\left(1-\frac{\nu_{\text {min }}}{\nu}\right) d \nu
\end{aligned}
$$

where $\sigma_{\nu}$ is the relevant photo-ionization cross section. $\mathrm{H}_{2}$ is the most important coolant for cooling the primordial gas, which leads to formation of the first stars. However, its hydrogen bond is weak and can be easily broken by photons in the $\mathrm{LW}$ bands between 11.2 and $13.6 \mathrm{eV}$. The small $\mathrm{H}_{2}$ fraction in the IGM creates only a little optical depth for LW photons, allowing them to propagate a much larger distance than ionizing photons. In our algorithm, self-shielding of $\mathrm{H}_{2}$ is not included because it is only important when $\mathrm{H}_{2}$ column densities are high. Here we treat the photodissociation of $\mathrm{H}_{2}$ in the optically thin limit and the dissociation rate in a volume constrained by causality within a radius, $r=c t$. The dissociation rate is given by $k_{\mathrm{H}_{2}}=1.1 \times 10^{8} \mathrm{FWW}_{\mathrm{LW}}{ }^{-1}$, where $F_{\mathrm{LW}}$ is the flux within LW bands (Greif et al. 2009a).

\subsection{X-ray Emission}

A compact binary may be able to produce radiative feedback in the form of X-rays. In this section, we describe the treatment of the radiation from such an X-ray binary source, if present. Our methodology is based on Jeon et al. (2012). In the local universe, the non-thermal emission spectrum from accreting back holes or neutron stars can be expressed as $F_{\nu} \propto \nu^{\beta}$, where $\beta=-1$ is the spectral index. More precisely, the full spectrum is a combination of a power-law component, representing nonthermal synchrotron radiation with the luminosity formula $L \sim \dot{M} c^{2}$, where $\dot{M}$ is the Bondi-Hoyle accretion model (Bondi \& Hoyle 1944), and a thermal multi-color disk component. We here ignore the latter, as we are only interested in the X-ray feedback on the general IGM, where only the optically thin, non-thermal photons contribute. We conservatively assume that the $\mathrm{BH}$ emission physics in the early universe is identical to the local case, and we therefore apply the same spectra for the $\mathrm{BH}$ originating from the first stars.

The propagation of high-energy photons is assumed to result in an isotropic radiation field, $\propto 1 / r^{2}$, which only depends on the distance from the $\mathrm{BH}$. The corresponding photo-ionization 
$\mathrm{S} 30+\mathrm{S} 30$
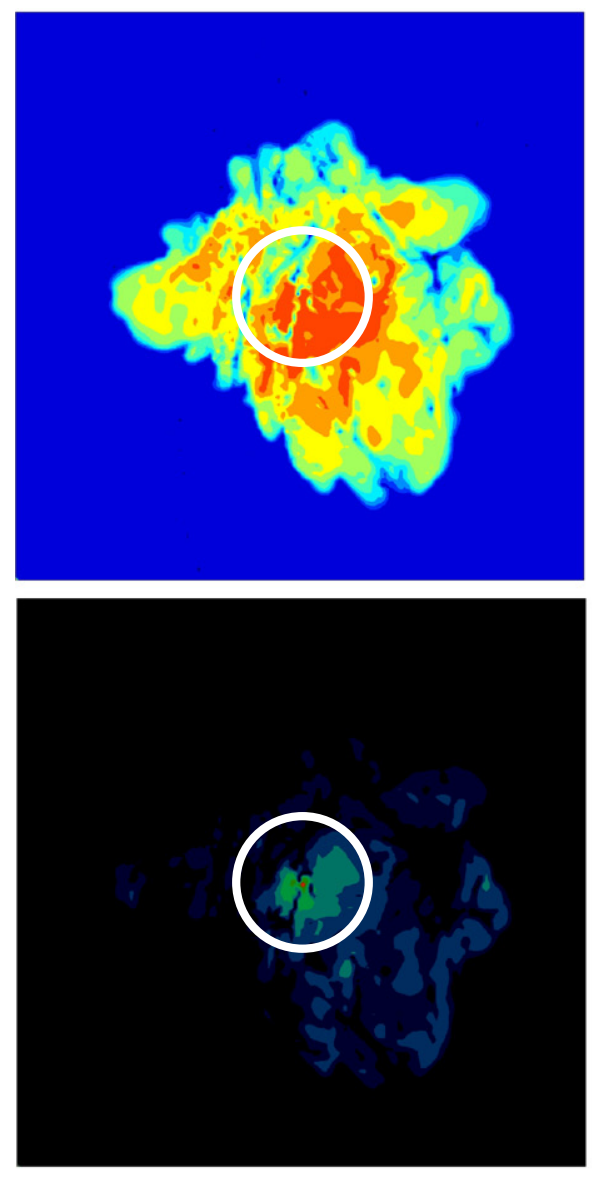

$\log \left(y\left[H^{+}\right]\right)$

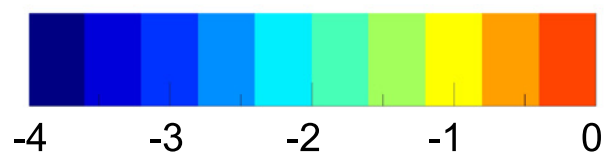

$\mathrm{S} 45+\mathrm{S} 15$
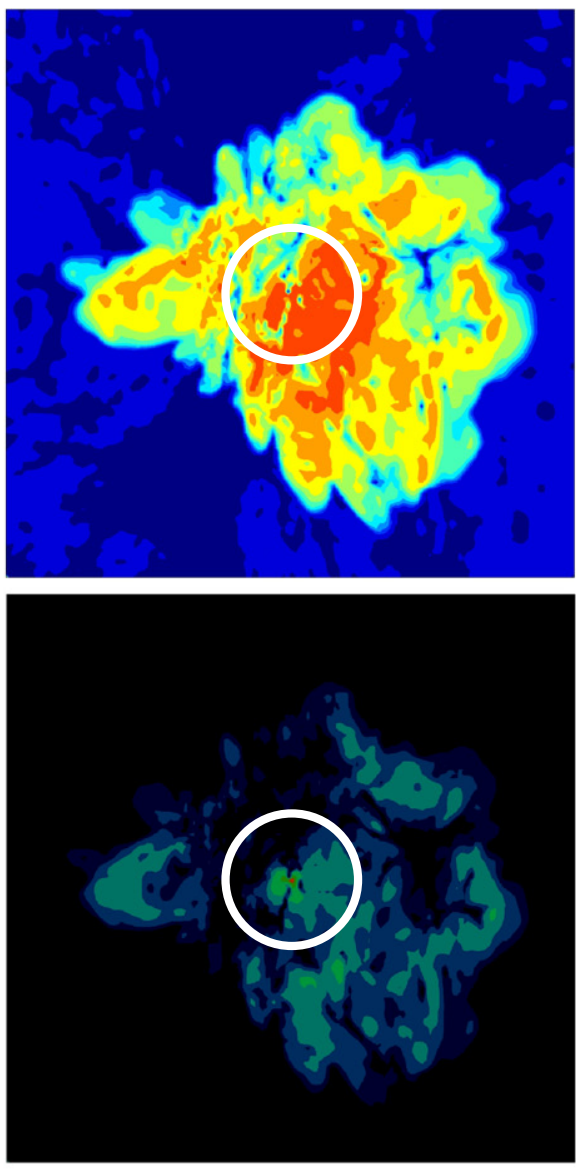

S60
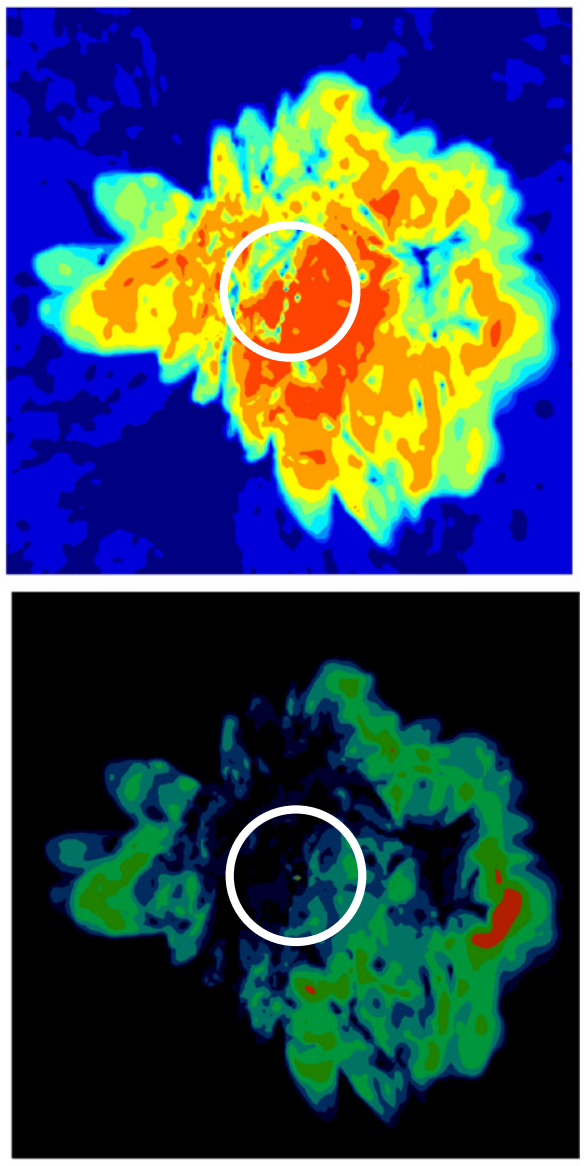

$\log \left(y\left[\mathrm{He}^{++}\right]\right)$

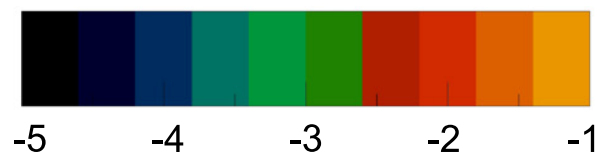

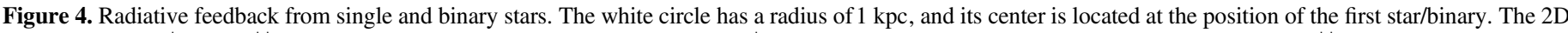

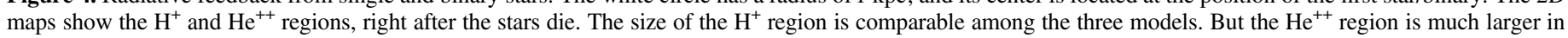
the S60 case.

and photo-heating rates can be written as (Jeon et al. 2012)

$$
k_{\text {ion }}=\frac{\dot{K}}{r_{\mathrm{pc}}^{2}}\left(\frac{\dot{M}_{\mathrm{BH}}}{10^{-6} M_{\odot} \mathrm{yr}^{-1}}\right),
$$

where

$$
\dot{K}=[1.96,2.48,0.49] \times 10^{-11} \mathrm{~s}^{-1},
$$

and

$$
\Gamma=\frac{n_{j} \dot{H}}{r_{\mathrm{pc}}^{2}}\left(\frac{\dot{M}_{\mathrm{BH}}}{10^{-6} M_{\odot} \mathrm{yr}^{-1}}\right)\left(1-f_{\mathrm{H} / \mathrm{He}}\right)
$$

Here

$$
\dot{H}=[7.81,9.43,1.63] \times 10^{-21} \mathrm{erg} \mathrm{cm}^{-3} \mathrm{~s}^{-1}
$$

for $\mathrm{HI}_{\mathrm{I}}, \mathrm{He} \mathrm{I}, \mathrm{He}$ II, respectively. $n_{j}$ is the corresponding number density, $r_{\mathrm{pc}}$ the distance from the star in units of pc, and $\dot{M}_{\mathrm{BH}}$ the mass accretion rate. Finally, $f_{\mathrm{H} / \mathrm{He}}$ are the fractions of the total photon energy expended in secondary ionizations (Shull \& van Steenberg 1985).

\subsection{SN Explosion and Metal Diffusion}

After several million years, the massive Pop III stars eventually exhaust their fuel, and many of them might have died as $\mathrm{SNe}$ or BHs. As we discussed above, the first SN explosions may be extremely powerful, accompanied by huge outputs of energy and metals. Here, we briefly discuss how we model SN explosions in our cosmological simulations.

When the star reaches the end of its lifetime, we initialize an SN blast wave by distributing the explosion energy among the SPH particles surrounding the sink that had marked the location of the Pop III star. Because the resolution of the simulation is about $1 \mathrm{pc}$, we cannot resolve individual $\mathrm{SNe}$ in both mass and space. Instead, we here select the particles within a region of $10 \mathrm{pc}$ to share the SN's thermal energy and metal yield. The gas within this region has mean temperatures about several million kelvin. On this scale, the blast wave is 


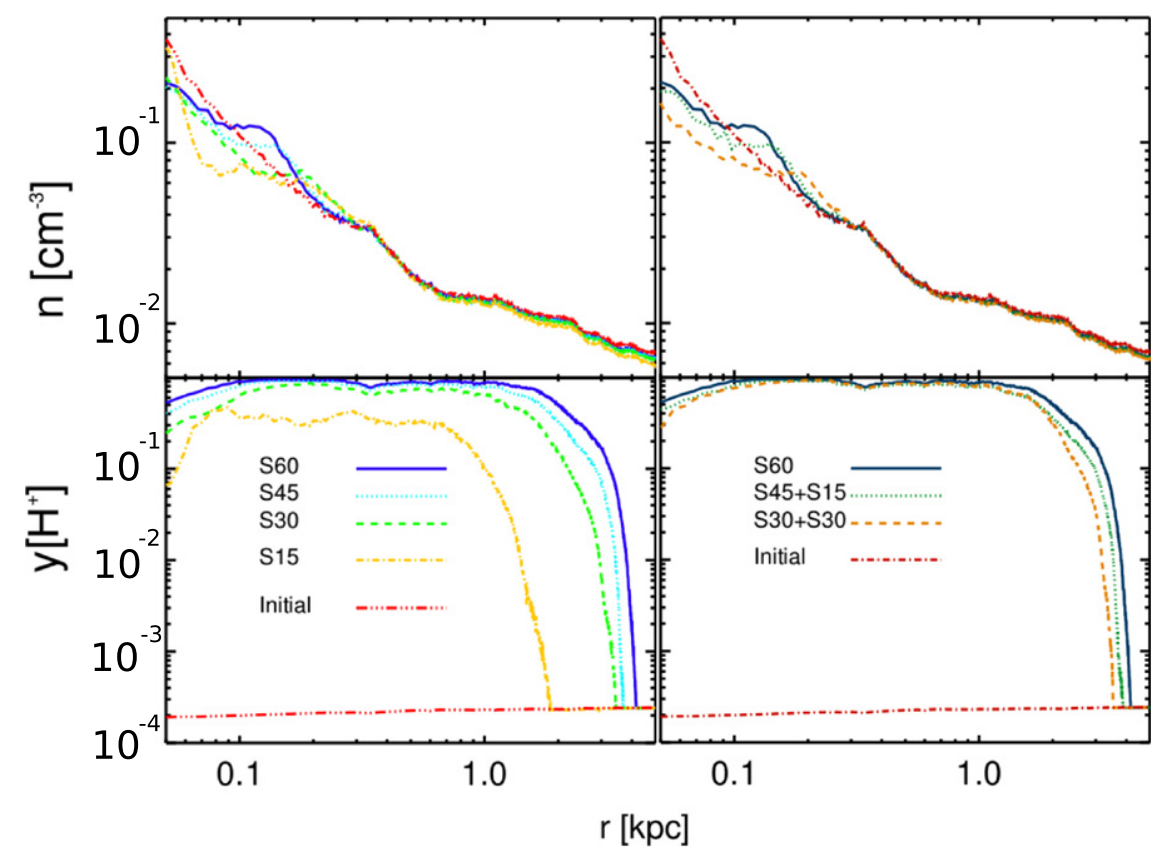

Figure 5. Density and ionization structure around the first star. The curves are mass-weighted profiles of gas density and the $\mathrm{H}^{+}$fraction inside the hot gas region created by the UV radiation. The red lines represent the conditions right before the birth of stars. There is a quick drop in $\mathrm{y}\left[\mathrm{H}^{+}\right]$, the fraction of ionized hydrogen, showing the boundaries between the photo-heated and unheated gas.

still close to its energy-conserving phase. The explosion energy of hypernovae ( $\mathrm{HNe})$ and pair-instability $\mathrm{SNe}$ can be up to $10^{52}-10^{53} \mathrm{erg}$, whereas a conventional core-collapse SN has about $10^{51} \mathrm{erg}$.

Mixing plays a crucial role in the transport of metals, which could be the most important coolant for subsequent star formation. We here cannot resolve the fine-grained mixing due to fluid instabilities in the early SN ejecta, developing on a scale far below $1 \mathrm{pc}$. However, we approximately model the coarse-grain mixing on cosmologically relevant timescales by applying the SPH diffusion scheme from Greif et al. (2009b). A precise treatment of the mixing of metals in cosmological simulations is not available so far because the turbulent motions responsible for mixing can cascade down to very small scales, far beyond the resolutions we can achieve now. We here, therefore, approximately model the effect of unresolved, subgrid turbulence as a diffusion process, linking the corresponding transport coefficients to the local physical conditions at the grid scale. For further algorithmic details, we refer the reader to Greif et al. (2009b).

After the $\mathrm{SN}$ explosion, metal cooling must be considered in the cooling network. We assume that $\mathrm{C}, \mathrm{O}$, and $\mathrm{Si}$ are produced with solar relative abundances, which are the dominant coolants for the gas contaminated by the first SNe. There are two distinct temperature regimes for these species. In low temperature gas, $T<2 \times 10^{4} \mathrm{~K}$, we use a chemical network presented in Glover \& Jappsen (2007), which follows the chemistry of $\mathrm{C}, \mathrm{C}^{+}, \mathrm{O}, \mathrm{O}^{+}, \mathrm{Si}, \mathrm{Si}^{+}$, and $\mathrm{Si}^{++}$, supplemental to the primordial species discussed above. This module considers the fine-structure cooling of $\mathrm{C}, \mathrm{C}^{+}, \mathrm{O}, \mathrm{Si}$, and $\mathrm{Si}^{+}$, whereas molecular cooling is not taken into account. At high temperatures, $T \geqslant 2 \times 10^{4} \mathrm{~K}$, due to the increasing number of ionization states, a full non-equilibrium treatment of metal chemistry becomes computationally prohibitive. Instead of directly solving the cooling network, we use the cooling rate table based on Sutherland \& Dopita (1993), which gives effective cooling rates for hydrogen and helium line cooling, as well as bremsstrahlung, at different metallicities. Dust cooling is not included because it would only become important at densities much higher than what is reached in our simulations.

\section{STELLAR MODELS}

\subsection{Single Star Models}

We use the Pop III stellar models of 15 (S15), 30 (S30), 45 (S45), and $60 M_{\odot}$ (S60) stars from the library of Heger \& Woosley (2010). These models are non-rotating stars and we assume no mass loss during the stellar evolution. We summarize key model characteristics in Table 1, where we distinguish the lifespan of main-sequence (MS; central hydrogen burning) and post-MS (until SN) evolution. The S15 model evolves in total for about $10.5 \mathrm{Myr}$ before encountering an iron core-collapse $\mathrm{SN}$ with an explosion energy of $1.2 \times 10^{51} \mathrm{erg}=1.2 \mathrm{~B}$ and a metal yield of $1.4 M_{\odot}$. Similarly, the S30, S45, and S60 models evolve for 5.7, 4.4, and 3.7 Myr, respectively. In assigning the final fate of our three most massive models, current understanding is still quite uncertain, and we here focus on a few illustrative possibilities. Specifically, in the cases of stars with masses of $30 M_{\odot}$ and above, we assume that they do not die as conventional iron core-collapse SNe. Instead, we assume that each of them either collapses into a $\mathrm{BH}$, triggering no explosion, or explodes as an HN with $10 \mathrm{~B}$ explosion energy, based on the collapsar model (Woosley 1993). When the star dies as a BH, all metals within the star fall back into the $\mathrm{BH}$, such that no enriched material is ejected. In the HN case, the S30, S45, and S60 models synthesized about $6.8,13.2$, and $20.6 M_{\odot}$ of heavy elements, and disperse them into the primordial IGM. We summarize the possible stellar fates in Table 2. For simplicity and to limit the number of cases in this study, we focus only on these simplified, limiting cases, and refer the reader to Heger \& 
$\mathrm{BH}$
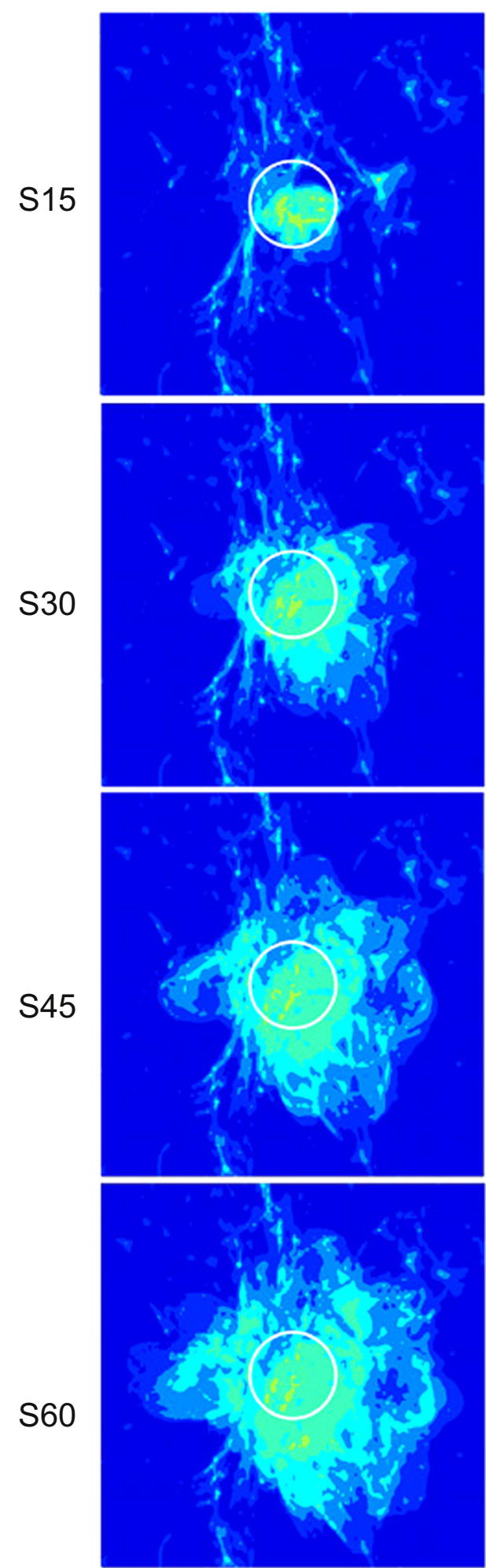

$\log (\mathrm{T} / \mathrm{K})$

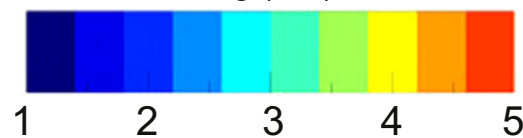

SN
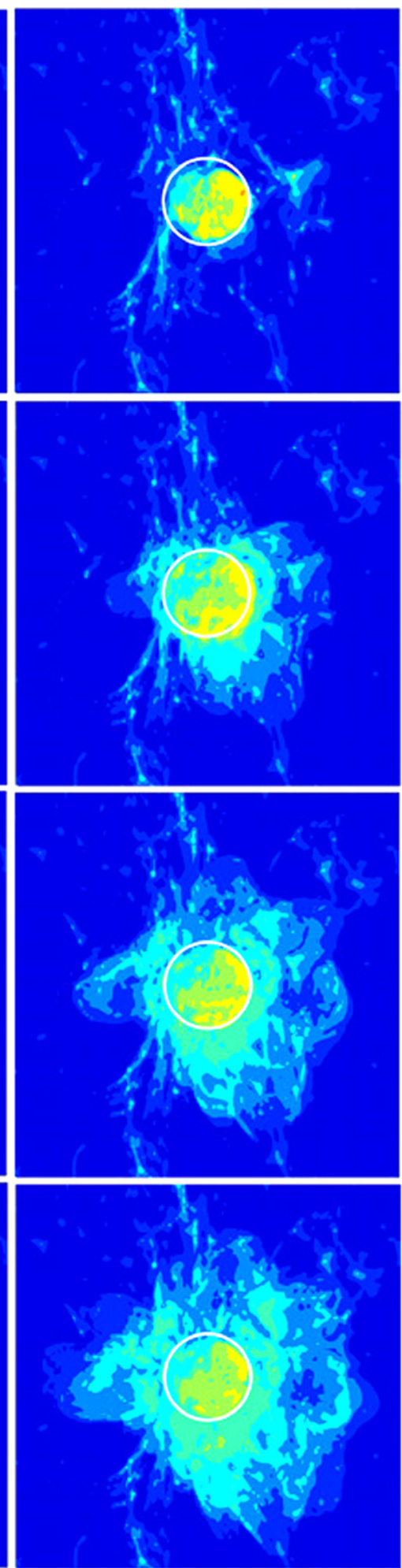

Metallicity
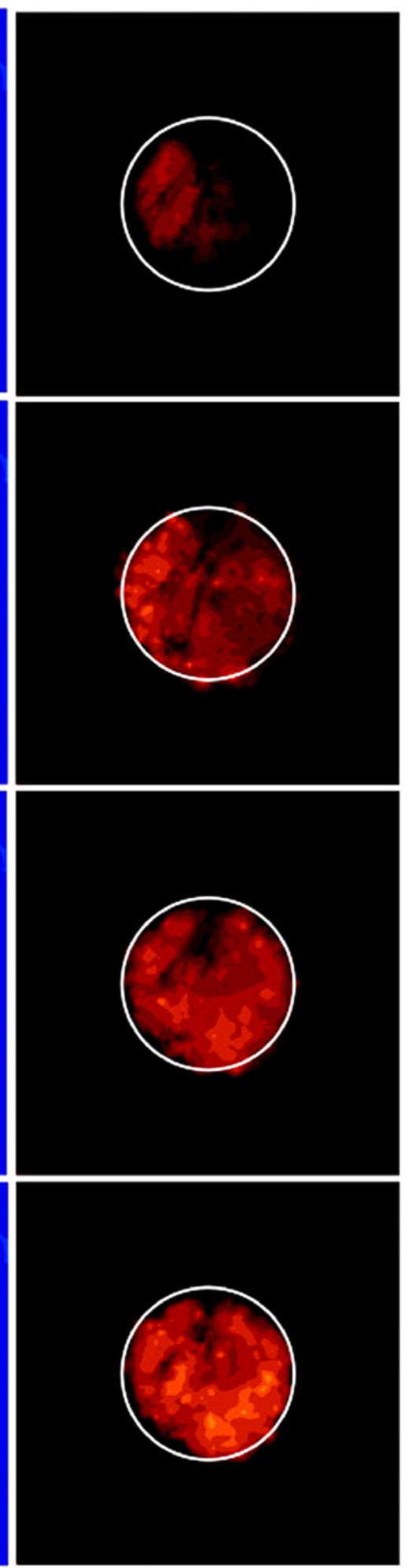

$\log \left(Z / Z_{\odot}\right)$

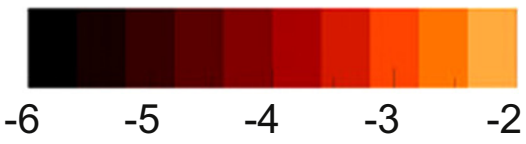

Figure 6. Comparison of the overall stellar feedback from all single stars at $15 \mathrm{Myr}$ after formation. The white circle has a radius of $1 \mathrm{kpc}$, and its center is located at the position of the first star. The SN ejecta stall at this time. If the stars die as SNe, the third-column panels show the resulting metallicity distribution. The white circles have the same meaning as in the previous figures. In the BH scenario, any radiation is simply shut down, and no further stellar feedback occurs. Without additional heating sources present, temperatures are significantly colder in the BH cases compared to the SN ones. The hypernova explosions experienced by the S30, S45, and S60 stars disperse the metals to $\sim 1 \mathrm{kpc}$, a much larger extent than what is achieved by the S15 star. 


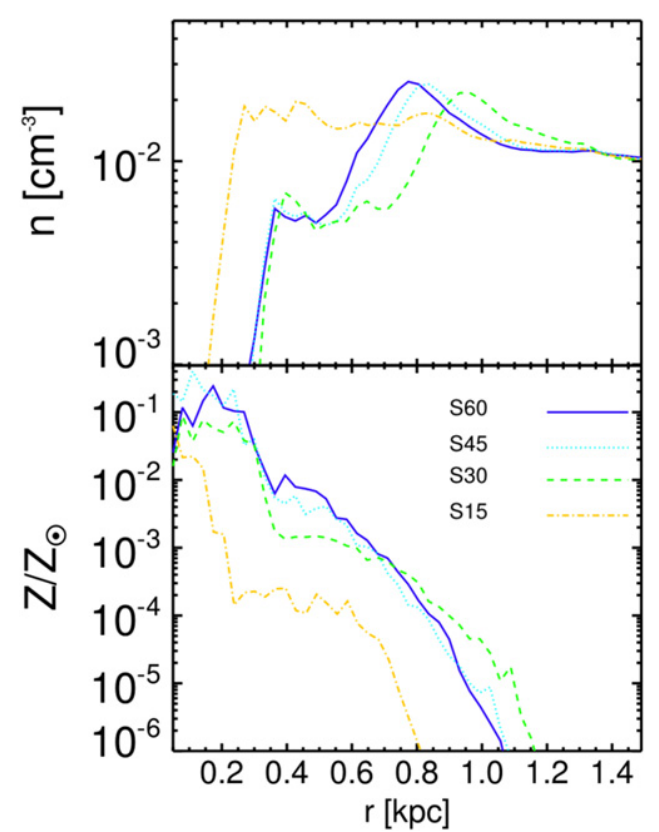

Figure 7. Strength of SN feedback from single stars. Shown are 1D gas density and metallicity profiles. The gas density inside the host halos has dropped to less than $0.01 \mathrm{~cm}^{-3}$. The ejected metal is mixed out to a radius of $\sim 1 \mathrm{kpc}$ for $\mathrm{S} 60$, S45, and S30, and $\sim 0.6 \mathrm{kpc}$ for $\mathrm{S} 15$.

Woosley (2010) for an extended discussion of Pop III SN models.

Massive Pop III stars are strong sources of UV radiation. Because of the predicted weak stellar winds from Pop III stars (Kudritzki 2002), there is no notable X-ray source contributing to the ionizing photon budget resulting from such winds. The UV radiation thus exclusively emerges from the hot stellar surface. Ionizing photon fluxes for all stellar models are given in Figure 1, where evolutionary effects are evident. Specifically, fluxes exhibit a gradual increase in the lower energy bands ( $\mathrm{LW}$ and $\mathrm{H}_{\mathrm{I}}$ ), and a decrease in the higher energy band (He II). The hydrogen-ionizing flux in the $\mathbf{S 6 0}$ model is about 10 times larger than for S15. The flux of more energetic photons is highly sensitive to stellar mass, such that for photons capable of ionizing He II, their ratio is 100:10:1 for S60:S30: S15. Because the lifespan of each star is different, we evaluate the overall ionizing power of an individual star by calculating the total amount of ionizing photons emitted before the star dies. As shown in Table 3, the S60 model produces about two, three, and six times more $\mathrm{HI}, \mathrm{He}$, and $\mathrm{He}$ II ionizing photons than S30. The cumulative ionizing power of S60 is also much stronger than the production from four S15 models combined. This implies that the overall radiative feedback of Pop III stars becomes weaker if their mass scale shrinks due to fragmentation.

Since our ray-tracing scheme cannot resolve a timescale less than a year, the radiation flash from the SN itself is not included here because the SN transit only lasts for about a few weeks to months. In principle, there could be a flash of hard radiation from the shock breakout that may eventually be observable (Scannapieco et al. 2005), but the total energy in this flash is small, due largely to the typically small radii of the Pop III stars at the time of death. Besides, the radiation from the subsequent main part of the SN light curve is largely at longer wavelengths and does not contribute much to the ionization. Our calculation shows that the total ionizing photon production during the SN is about $10^{-5}$ that of the MS phase. Furthermore, most of the SN explosion energy goes into the thermal and kinetic energy of the ejecta (de Souza et al. 2013; Whalen et al. 2014).

\subsection{Binary Star Models}

The ubiquitous fragmentation of primordial star-forming clouds allows the widespread formation of binary stars. We consider binary stars with a total mass of $60 M_{\odot}$, specifically a system with two stars of equal mass (mass ratio 1:1) and another one with a mass ratio of 3:1, in accordance with current theoretical understanding. Shu et al. (1987) and Larson (2003) suggest that binary stars with (close to) equal mass are common in the local universe. Our binary models thus contain both asymmetric cases of $45 M_{\odot}+15 M_{\odot}$ Pop III stars and symmetric ones of $30 M_{\odot}+30 M_{\odot}$ Pop III stars. To keep the binary models simple, we do not consider binary star mergers, and neglect any mass ejection as an idealized approximation. We are aware of and advise the reader of the shortcomings of these simplifications, compared to more realistic binary models (Langer 2012). Below, we discuss the select binary scenarios considered here (see Table 4).

1. $S 30+S 30(H N)$ : This model contains two $30 M_{\odot}$ Pop III stars, each represented by our $\mathrm{S} 30$ model. We assume that both stars form at the same time and evolve together for about 5.7 Myr, after which they both die as an $\mathrm{HN}$.

2. $S 30+S 30(B H)$ : This scenario is very similar to $\mathrm{S} 30+\mathrm{S} 30$ $(\mathrm{HN})$, but now both stars directly collapse into BHs, without triggering an $\mathrm{SN}$.

3. $\$ 45+S 15(B H)$ : The binary contains two stars, represented by S45 and S15 models. Both of them form at the same time and evolve together for $4.4 \mathrm{Myr}$. Subsequently, the $\mathrm{S} 45$ component dies as a $\mathrm{BH}$, and the system becomes an X-ray binary source due to the transfer of mass from the S15 companion onto the $\mathrm{BH}$. We approximately assume that the entire mass of the primary collapses into the $\mathrm{BH}$, and that the system remains bound. We employ a mass transfer rate of $10^{-6} M_{\odot} \mathrm{yr}^{-1}$, active for about $10 \mathrm{Myr}$. Because the S15 secondary would lose much of its mass during this X-ray binary phase, we assume a white dwarf (WD) death, without an SN explosion.

4. $S 45+S 15(H N)$ : Both stars again form at the same time and evolve together for $4.4 \mathrm{Myr}$, then the S45 primary dies as an HN. The S15 component evolves for another $6 \mathrm{Myr}$, then dies as a CCSN. For a wide binary, the kick velocity is small, and the star will die essentially at the location of the original binary. However, in a close binary situation, the orbital velocity is high, and the S15 secondary would acquire a kick of about $100 \mathrm{~km} \mathrm{~s}^{-1}$, assuming a binary separation of about 1 AU. Such a velocity might allow the star to travel close to $1 \mathrm{kpc}$ before dying as an SN. In this case, the ejected S15 model could become a moving radiation source and disperse its metal production from a site quite remote from where it formed. Conroy \& Kratter (2012) suggested that the runaway massive stars could also contribute to the reionization of the universe. For simplicity, we do not explore this intriguing scenario in this paper. 

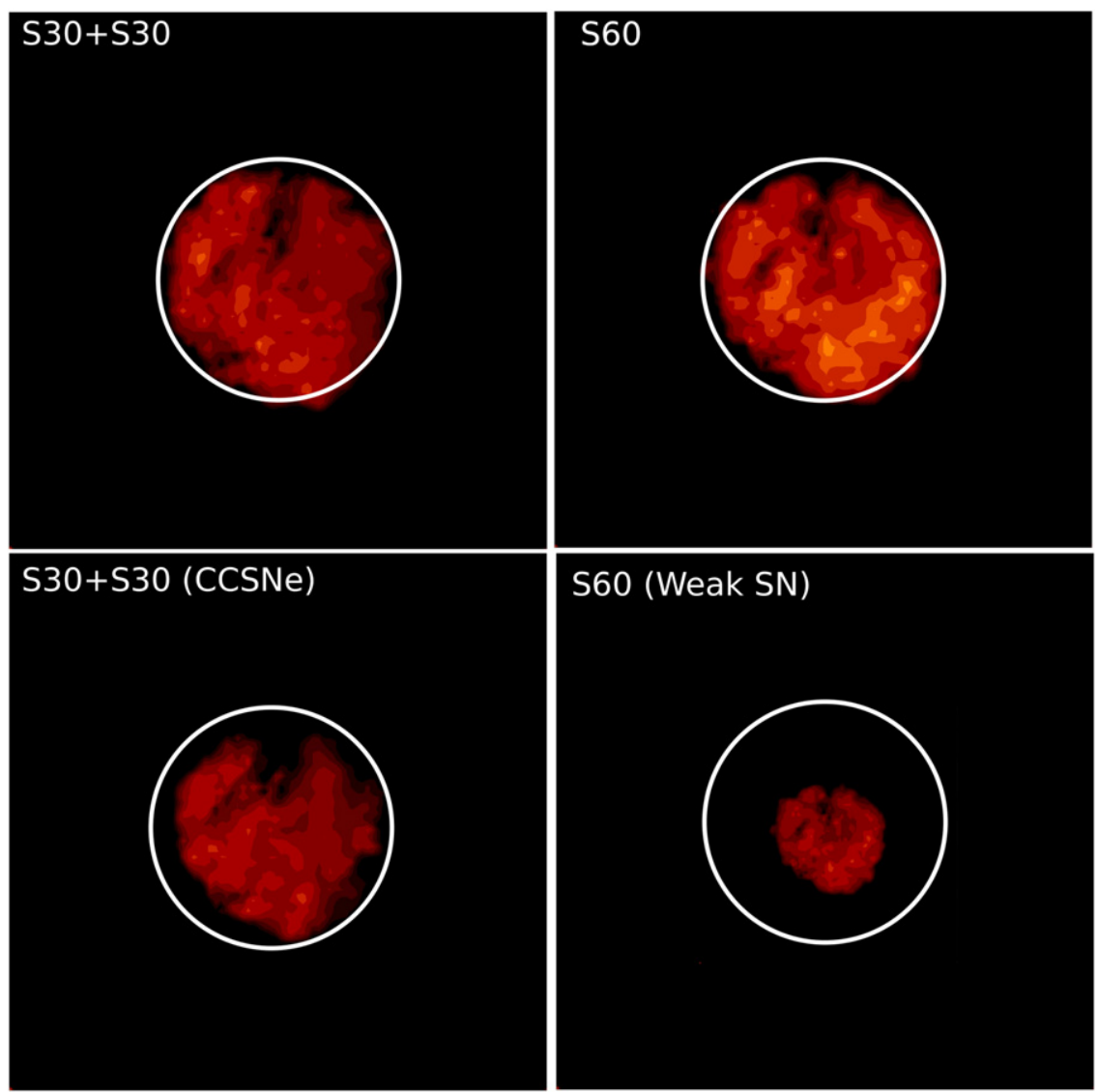

$\log \left(Z / Z_{\odot}\right)$

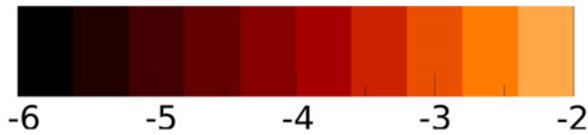

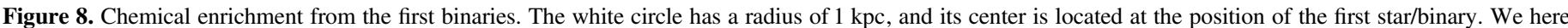

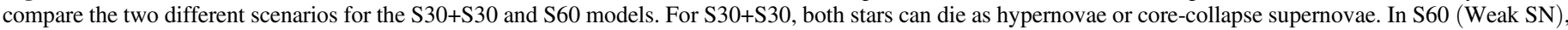

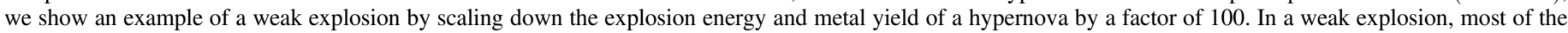
metals fall back onto the $\mathrm{BH}$ without being ejected.

Since we are considering only non-interacting cases, their resulting UV flux can easily be obtained by summing over the individual contributions from the two component stars. Figure 2 shows the ionizing photon flux of the S45+S15, S30+S30, and S60 models, respectively, and Table 5 lists the total number of ionizing photons emitted. The UV fluxes of the three models are comparable within a factor of two. However, a single star, S60, still produces stronger flux than binary systems with the same overall mass. Differences are largest in the amount of ionizing He II photons, as this rate is extremely sensitive to the mass of the primary.

\section{RESULTS}

The impact of the first binary stars on the early universe can be divided into three different classes; UV radiation, SN, and $\mathrm{X}$-ray. The UV-radiative feedback here refers to the soft (LW) and hard (ionizing) photons produced by the binaries during their stellar evolution. To facilitate convenient comparison, we also briefly discuss the feedback from single Pop III stars, referring the reader to the extensive literature on this topic for further detail. We note that our single star models improve on earlier treatments by including some key features, such as the realistic modeling of the time dependence of the UV fluxes in response to the underlying stellar evolution. We present the results from our cosmological simulations following the chronologically order of how the first single or binary stars evolve: birth, evolution, and demise. Because the results contain many cosmological simulations of different feedback models, we summarize the stellar models, their feedbacks, and associated results in Table 6.

\subsection{Radiative Feedback}

The first stellar system forms inside a minihalo, with a total mass of about $10^{6} M_{\odot}$ and a virial radius of $\sim 100 \mathrm{pc}$, located in the region with the highest mass resolution at $z \sim 27$. This allows us to resolve key small-scale features of the ensuing stellar feedback. Once the gas density inside the star-forming cloud exceeds the threshold for sink creation, either a single star or a binary system is assumed to promptly form. By using sink particles, the realistic assembly history of a protostar via an extended phase of accretion is not modelled, which still is computationally prohibitive. Instead, we assume that the sink particles immediately represent fully developed Pop III stars, acting as sources of UV radiation. The gas inside the halo is 
rapidly photo-heated up to temperatures of $T \approx 2 \times 10^{4} \mathrm{~K}$. This drives the sound speed up to $30 \mathrm{~km} \mathrm{~s}^{-1}$, whereas the escape velocity of the host halo is only about $3 \mathrm{~km} \mathrm{~s}^{-1}$. The photo-heated gas is thus blown out of the shallow potential well of the minihalo. UV photons not only heat up the gas but also ionize the neutral hydrogen and helium. The ionization-front (Ifront) propagation begins with a short supersonic phase (Rtype), then switches to a subsonic phase (D-type), because the I-front is trapped behind a hydrodynamical shock (e.g. Glover \& Brand 2001; Whalen et al. 2004, 2010). The I-front

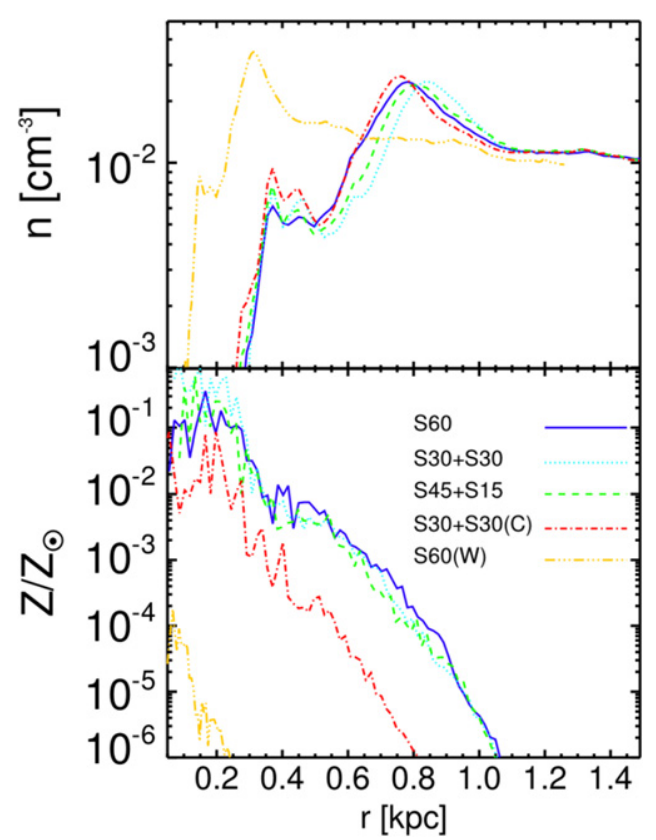

Figure 9. $\mathrm{SN}$ feedback from single and binary stars. The two panels show 1D gas density and metallicity profiles inside the SN ejecta. S60, S30+S30, and $\mathrm{S} 45+\mathrm{S} 15$ are hypernova explosions. $\mathrm{S} 30+\mathrm{S} 30(\mathrm{C})$ is a core-collapse $\mathrm{SN}$, and $\mathrm{S} 60(\mathrm{~W})$ is a weak $\mathrm{SN}$. eventually breaks out, jumping ahead of the shock, and supersonically propagates into the low-density IGM.

Since the lifetime of the individual stars is different, we compare their radiative feedback when they die and their UV radiation is terminated. We first show the resulting gas temperatures around the host minihalo in Figure 3. A giant bubble of hot, ionized, gas is created around the central star, with an inner region that has reached temperatures in excess of $10^{4} \mathrm{~K}$. The shapes of these bubbles are very irregular due to the inhomogeneous and anisotropic distribution of the gas in the surrounding IGM. The bubble sizes reflect the strength of the UV emission rates, which highly depend on stellar mass. Specifically, our S60 model creates the largest bubble with a size of about $5 \mathrm{kpc}$. The $\mathrm{S} 15$ model, on the other hand, only gives rise to an ionized region with a size of $\sim 2 \mathrm{kpc}$, and a much cooler gas temperature.

For binary stars, we compare cases of equal total stellar mass in Figure 4. Overall bubble sizes for the equal-mass models are comparable. However, there is a significant difference in the resulting $\mathrm{He}^{++}$regions. Ionizing $\mathrm{He}^{+}$requires photon energies of $h \nu>54.4 \mathrm{eV}$, four times higher than the threshold to ionize neutral hydrogen. Here, it greatly matters how the available stellar mass is divided among the individual components, such that the S60 model exhibits significantly larger $\mathrm{He}^{+}$ionizing rates than the binary models of equal mass, S30+S30, and S45 $+\mathrm{S} 15$. This difference may be reflected in the strength of the $\mathrm{He}^{+}$recombination line at $1640 \AA$, providing a distinctive signature to distinguish between Pop III single and binary systems, since the latter create much smaller $\mathrm{He}^{++}$regions, for a fixed total stellar mass.

To better evaluate the impact of the radiative feedback, we map the three-dimensional structure of the hot bubbles onto 1D radial profiles in Figure 5. We first discuss the gas density profile. Due to the UV photo-heating, the gas density in the center of the minihalo has dropped to $0.2 \mathrm{~cm}^{-3}$ at the end of star's lifetime. The baryonic outflow extends to a radius of $150 \sim 200 \mathrm{pc}$, slightly larger than the virial radius of the host halo, such that any subsequent star formation is suppressed by
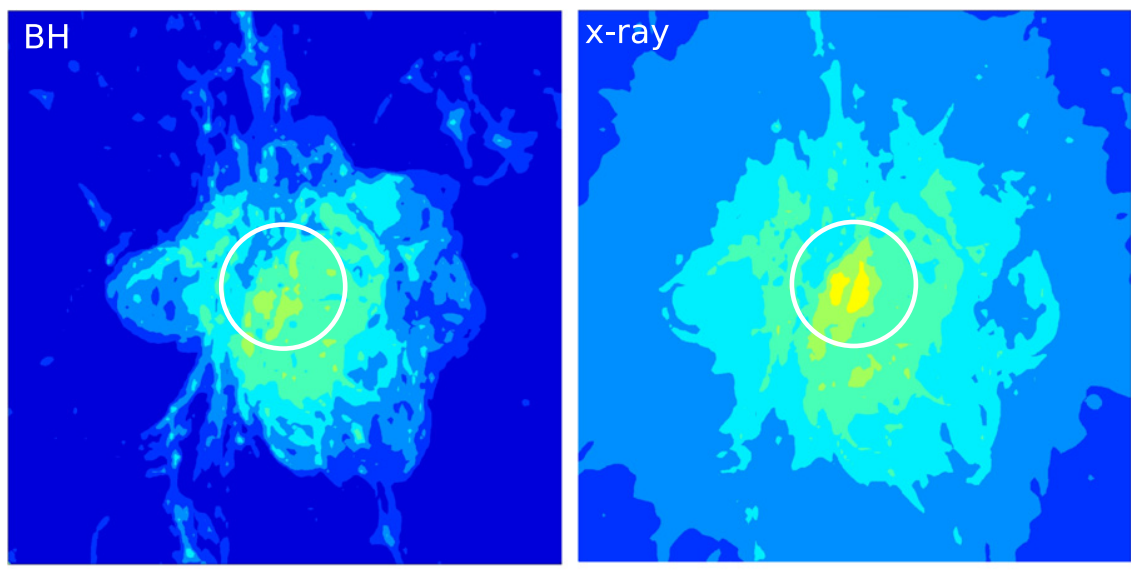

$\log (\mathrm{T} / \mathrm{K})$

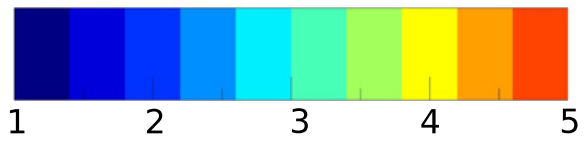

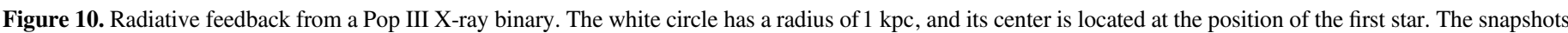

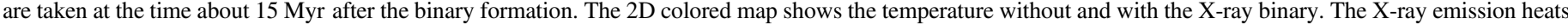
up the relic $\mathrm{H}$ II region and beyond. The morphology of the feedback is rather isotropic and homogeneous. 


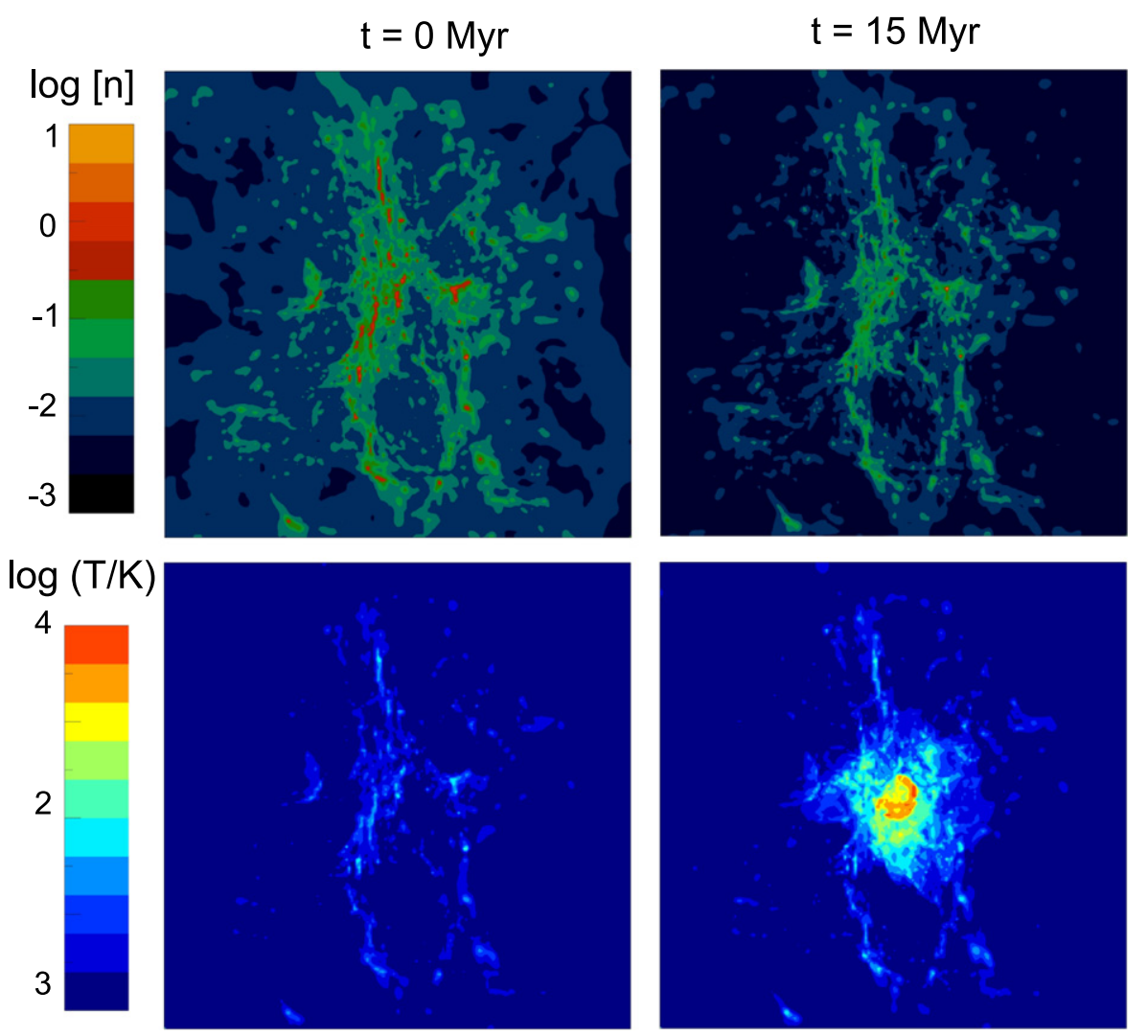

Figure 11. Cosmological impact of a $60 M_{\odot}$ star. Panels show temperatures and densities before/after the stellar feedback from a $60 M_{\odot}$ star. The left two panels are at $t=0$, right before the star formation and the right two panels are at $t=15 \mathrm{Myr}$. The size of each panel is about $10 \times 10 \mathrm{kpc}^{2}$. Some higher density clumps have been smoothed due to the radiative and SN feedback, which also chemically enriches the pristine gas within the orange circle of radius $\sim 1 \mathrm{kpc}$. Both radiative and supernova feedback heat up the gas and change its chemistry on a scale of 3-4 kpc.

expelling the gas through this photo-evaporation. Besides their hydrodynamic feedback, UV photons also affect the chemistry of the primordial gas by changing its ionization state, and releasing free electrons that can catalyze $\mathrm{H}_{2}$ formation. The weaker UV emission of the S15 model results in a relatively smaller $\mathrm{H}^{+}$region, whereas those of $\mathrm{S} 60, \mathrm{~S} 45$, and $\mathrm{S} 30$ have radii close to $2 \mathrm{kpc}$. The difference in the central gas density profile is mainly due to the duration over which photo-heating is active. Binary models exhibit longer overall lifetimes, allowing the gas to escape farther into the IGM, such that the resulting gas densities within the halo are lower. It is not clear whether the UV radiation can penetrate into nearby minihalos and affect their star formation or not. When the stars die, and if there are no additional heating sources, the ionized gas will cool and then recombine, eventually extinguishing the fossil $\mathrm{H}^{+}$ regions. The relevant timescales can be estimated as follows (Bromm et al. 2002). The cooling time of primordial gas is approximately $t_{\text {cool }} \approx n k_{B} T / \Lambda \approx 10^{5} \sim 10^{6} \mathrm{yr}$, where $\Lambda \propto n^{2}$ is the cooling rate, and $k_{B}$ the Boltzmann constant. For the recombination timescale, we estimate $t_{\text {rec }} \approx\left(k_{\text {rec }} n\right)^{-1} \approx 10^{6} \sim 10^{7} \mathrm{yr}$, where the recombination coefficient is $k_{\text {rec }} \approx 10^{-12} \mathrm{~cm}^{3} \mathrm{~s}^{-1}$, and the IGM densities $n \approx 0.01 \mathrm{~cm}^{-3}$.

\subsection{SN Feedback}

The majority of Pop III stars may finally die as $\mathrm{SNe}$ or directly collapse into BHs. In this section, we discuss the SN feedback from single and binary stars. When the stars die, we assume for simplicity that their UV radiation is immediately shut off. We employ the SN explosion energies and metal yields discussed in Section 3, depending on the properties of the progenitor star. The $\mathrm{SN}$ explosion creates a strong shock wave, traveling with a velocity of $v_{\mathrm{sn}} \approx 10^{4} \mathrm{~km} \mathrm{~s}^{-1}$. The energy carried by the shock is able to reheat the relic $\mathrm{H}$ II region for an additional $t_{\mathrm{sn}} \approx r_{h} / V_{\mathrm{sn}} \approx 0.4 \mathrm{Myr}$, assuming $\mathrm{H}$ II region radii of $r_{h} \approx 4 \mathrm{kpc}$. The shock heating in the simulation is roughly about $0.6 \mathrm{Myr}$ because the shock velocity is slowed down due to radiative cooling. After the shock dissipates, a hot and metalrich bubble is left behind in the IGM. This bubble continues to expand for about another 5 million years, with an increasingly ill-defined boundary. Eventually, the thermal energy of the initial ejecta is radiated away, and the expansion stalls. The mixing of the metals with primordial gas predominantly occurs before stalling. On the other hand, if a single star directly dies as a BH, no feedback is taken into account (but see Section 4.3).

We first discuss the combined feedback from UV radiation and $\mathrm{SNe} / \mathrm{BHs}$, evaluated $15 \mathrm{Myr}$ after the birth of the stars, in Figure 6. At this moment, the SN blast wave has stalled. The heating from the hot $\mathrm{SN}$ ejecta maintains elevated ionization in the central part of the $\mathrm{H}$ II region. The chemical feedback of the S15 model is initiated by a CCSN with explosion energy of about $1.2 \mathrm{~B}$ and an ejected metal mass of $1.4 M_{\odot}$. These metals are dispersed out to a radius of $\sim 0.5 \mathrm{kpc}$, resulting in an average metallicity of $\sim 10^{-5}-10^{-4} Z_{\odot}$. According to our stellar model assumptions, the S30, S45, and S60 cases all die as an $\mathrm{HN}$, dispersing $6.9,13.3$, and $20.7 M_{\odot}$ of heavy elements, 
respectively. For the $\mathrm{HN}$ cases, the metal-enriched bubbles have reached radial sizes of $\sim 1 \mathrm{kpc}$ with corresponding metallicities of $10^{-4}-10^{-2} Z_{\odot}$. Although all $\mathrm{HN}$ cases exhibit a similar overall range of chemical enrichment, there are noticeable differences in the detailed distribution of metals. These differences in turn may reflect how the preceeding radiative feedback from the different model stars has shaped the gas distribution inside the IGM, which could affect the transport of metals later on. To further facilitate the comparison between the single-star cases, we present 1D profiles of gas density and metallicity in Figure 7 . The strong SN blast waves in all cases substantially suppress the gas density in the host halo, resulting in $n<0.01 \mathrm{~g} \mathrm{~cm}^{-3}$, similar to that of the background IGM at this redshift of $z \sim 26$. The extent of the central void is about $200 \mathrm{pc}$ for the CCSN case (S15), and $500 \mathrm{pc}$ for the HN cases, respectively. The metallicity profiles for the $\mathrm{HN}$ cases are quite similar, but the S30 model enriches the IGM out to a slightly larger radius in response to its higher blast wave velocity. Finally, instead of SN explosions, the stars may directly collapse into a $\mathrm{BH}$, a possibility which becomes increasingly likely with increasing progenitor mass. For such a $\mathrm{BH}$ fate, the relic $\mathrm{H}$ in regions would quickly begin to cool to about $10^{3} \mathrm{~K}$, and recombination would suppress the abundance of free electrons.

We now discuss the SN feedback from binary stars. To enable a meaningful comparison, we fix the total mass in Pop III stars, here $60 M_{\odot}$, either locked up in a single star or distributed among binary partners $(\mathrm{S} 30+\mathrm{S} 30$ or $\mathrm{S} 45+\mathrm{S} 15)$. The key question then is whether or not there are significant differences. Stellar evolution models strongly suggest that $60-80 M_{\odot}$ Pop III stars are likely to die as BHs, possibly accompanied by very weak SN explosions. Hence, we also consider the case of a $60 M_{\odot}$ star dying as such a weak SN with an explosion energy of $\sim 0.1 \mathrm{~B}$ and a metal yield of $\sim 0.1 \mathrm{M}_{\odot}$, due to strong fall back during the BH-forming explosion. The basic trends can be gleaned from Figures 8 and 9. As long as at least one component explodes as an $\mathrm{HN}$, the resulting metal enrichment is very similar. However, the enrichment from the binary systems is more robust, in the sense that the single S60 star is likely to experience only a weak explosion accompanied by much reduced heavy element production and dispersal. Thus, the recent revision of the Pop III star formation paradigm away from a single-star outcome to ubiquitous binarity in effect enhances chemical feedback in the early universe.

\subsection{X-ray Feedback}

One particularly interesting variant of the $\mathrm{S} 45+\mathrm{S} 15$ model is the presence of long-lived X-ray feedback. Because of the uncertainty in the stellar evolution model, the $45 M_{\odot}$ star can possibly collapse into a $\mathrm{BH}$ instead of blowing up as an SN. In the case of a close binary, mass transfer from the $15 M_{\odot}$ companion, still alive at this time, becomes possible. Mass accretion onto the compact object can efficiently extract the gravitational energy of the infalling material, converting it into the thermal energy within the accretion disk that leads to X-ray emission. Unlike the ionizing photons from stars, the X-rays can more easily penetrate the IGM because of the much reduced opacity at high energies (e.g., Machacek et al. 2003; Kuhlen \& Madau 2005; Jeon et al. 2012). Here, we assume a constant accretion rate onto the central $\mathrm{BH}$ of about $10^{-6} M_{\odot} \mathrm{yr}^{-1}$, which lasts for about 10 million years. For simplicity, we further assume that the $15 M_{\odot}$ companion star eventually dies as a WD without an SN explosion, and we neglect its UV radiation during the accretion phase. We show the impact of such a Pop III X-ray binary, comparing it with the non-X-ray case, in Figure 10. In the absence of X-ray emission, the temperature of the the relic $\mathrm{H}$ II region quickly declines and the ionized hydrogen recombines. An active X-ray binary, on the other hand, provides a prolonged heating source, maintaining the temperature and ionization inside the relic $\mathrm{H}_{\text {II }}$ region, and further heating up the gas beyond its boundary, up to several hundred kelvin. More importantly, the X-ray emission can change the free electron fraction in the IGM, which is critical for $\mathrm{H}_{2}$ formation. Because the IGM is optically thin to $\mathrm{X}$-ray photons, the resulting heating is quite homogeneous and isotropic. We also find that the X-ray photons may penetrate the gas of nearby halos, thus affecting their star formation properties.

\section{DISCUSSIONS AND CONCLUSIONS}

We have presented the results from cosmological simulations of the impact of Pop III stars, specifically focusing on the new effects arising from the presence of binaries. We improve on earlier simulations by using updated Pop III stellar models. In ascertaining the cosmological impact of the first binaries, we consider their radiative, SN, and X-ray feedback. By comparing a single $60 M_{\odot}$ star and the corresponding binary systems with equal total mass, we find that the resulting numbers of hydrogen-ionizing photons are very similar. However, for $\mathrm{He}^{+}$ ionizing radiation, the binary stars are significantly weaker than the single star because the more energetic UV photon production is strongly reduced in the less massive stars. If binary stars thus were the typical outcome of Pop III star formation in minihalos, detection of the distinct emission lines from $\mathrm{He}^{++}$recombination, most prominently the $1640 \AA$ line, would be very challenging. Because the X-ray feedback strongly depends on the spectrum of X-ray binaries, which is uncertain in the high redshifts, its observational signature is more difficult to predict. Recently, Xu et al. (2014) and Ahn et al. (2014) suggested that the X-ray feedback of the Pop III binaries can be examined by the $21 \mathrm{~cm}$ observations.

We here explore cases where the stars die as core-collapse $\mathrm{SNe}$ or as hypernovae. In all cases, the mechanical feedback from the explosions expels the gas from the host systems, thus suppressing any subsequent star formation in the same halo, at least for of order $10 \mathrm{Myr}$. To trigger any further star formation, the expelled gas needs to be driven back to high density, possibly as a result of halo mergers within bottom-up structure formation later on. For a $15 M_{\odot}$ star, the expected final fate is a core-collapse SN, whereas the fate of 30,45 , and $60 M_{\odot}$ Pop III stars is still poorly understood. To bracket parameter space, we here assume that they die as energetic hypernovae, weak SNe, or directly collapse into BHs. It is evident that for such more massive progenitors, chemical feedback can span a broad range of possibilities. The most effective feedback is provided by the hypernova models, where the nearby IGM is typically enriched to average metallicities of $\sim 10^{-4}-10^{-3} Z_{\odot}$, out to $\sim 2 \mathrm{kpc}$.

Even single, energetic SNe can impact the early universe on cosmological scales. We demonstrate this in Figure 11, showing the feedback from a single $60 M_{\odot}$ star, undergoing an $\mathrm{HN}$ explosion. The resulting feedback significantly enhances the IGM temperature, smoothes out density structures 
in nearby halos, and enriches the primordial gas over regions of $\sim 2 \mathrm{kpc}$. However, the formation of a single massive star inside a minihalo increases the probability of collapsing into a $\mathrm{BH}$ without any chemical enrichment. On the other hand, binary star formation greatly buttresses the likelihood that metal enrichment will occur. In effect, binary formation is much less likely to keep the early universe metal-free, with consequences for the prompt transition in star formation mode to the lowmass-dominated Population II.

Realistic binary stellar models could introduce a very rich phenomenology of evolutionary pathways. Among them are ejection scenarios, where one component is flung out, such that it may explode in the outskirts of its host halo, as opposed to the location of its birth, as implicitly assumed here. There is also rich physics related to the early evolution of the $\mathrm{SN}$ remnant, when shock breakout occurs, and hydrodynamical mixing takes place. All of these intriguing aspects of binary progenitors will be explored in future simulations, with greatly improved spatial and temporal resolution. The exploratory models presented here, however, already clearly indicate the importance of studying binary-related feedback in the early universe. The imprint from the violent death of Pop III stars, in all its variety, might soon be amenable to empirical testing with the James Webb Space Telescope, to be launched around 2018. One key aspect of this search will be to distinguish the possible signature of binarity in primordial star formation.

The authors thank the anonymous referee for many constructive suggestions. We also thank Jarrett Johnson, Dan Whalen, and Ryan Cooke for many useful discussions. K.C. was supported by an IAU-Gruber Fellowship, a Stanwood Johnston Fellowship, and a KITP Graduate Fellowship. Work at UCSC has been supported by DOE HEP Program under contract DE-SC0010676; the NSF (AST 0909129), and the NASA Theory Program (NNX14AH34G). V.B. acknowledges support from NSF grant AST-1009928 and NASA grant NNX09AJ33G. A.H. acknowledges support by an ARC Future Fellowship (FT120100363) and a Monash University Larkins Fellowship. This work has been supported by the DOE grants; DE-GF02-87ER40328, DE-FC02-09ER41618, and by the NSF grants AST-1109394, and PHY02-16783. All numerical simulations were performed with allocations from the University of Minnesota Supercomputing Institute and the National Energy Research Scientific Computing Center.

\section{REFERENCES}

Abel, T., Bryan, G. L., \& Norman, M. L. 2002, Sci, 295, 93 Abel, T., Wise, J. H., \& Bryan, G. L. 2007, ApJL, 659, L87

Ahn, K., Xu, H., Norman, M. L., Alvarez, M. A., \& Wise, J. H. 2014, arXiv: 1405.2085

Alvarez, M. A., Bromm, V., \& Shapiro, P. R. 2006, ApJ, 639, 621

Alvarez, M. A., Wise, J. H., \& Abel, T. 2009, ApJL, 701, L133

Barkana, R., \& Loeb, A. 2001, PhR, 349, 125

Barkat, Z., Rakavy, G., \& Sack, N. 1967, PRL, 18, 379

Beers, T. C., \& Christlieb, N. 2005, ARA\&A, 43, 531

Bond, J. R., Arnett, W. D., \& Carr, B. J. 1984, ApJ, 280, 825

Bondi, H., \& Hoyle, F. 1944, MNRAS, 104, 273

Bromm, V. 2013, RPPh, 76, 112901

Bromm, V., Coppi, P. S., \& Larson, R. B. 1999, ApJL, 527, L5

Bromm, V., Coppi, P. S., \& Larson, R. B. 2002, ApJ, 564, 23

Bromm, V., Ferrara, A., Coppi, P. S., \& Larson, R. B. 2001a, MNRAS, 328,969

Bromm, V., Kudritzki, R. P., \& Loeb, A. 2001b, ApJ, 552, 464

Bromm, V., \& Larson, R. B. 2004, ARA\&A, 42, 79
Bromm, V., \& Yoshida, N. 2011, ARA\&A, 49, 373

Bromm, V., Yoshida, N., Hernquist, L., \& McKee, C. F. 2009, Natur, 459, 49 Chatzopoulos, E., \& Wheeler, J. C. 2012, ApJ, 748, 42

Chen, K.-J., Heger, A., Woosley, S., Almgren, A., \& Whalen, D. J. 2014, ApJ, 792, 44

Ciardi, B., \& Ferrara, A. 2005, SSRv, 116, 625

Conroy, C., \& Kratter, K. M. 2012, ApJ, 755, 123

Couchman, H. M. P., \& Rees, M. J. 1986, MNRAS, 221, 53

de Souza, R. S., Ishida, E. E. O., Johnson, J. L., Whalen, D. J., \& Mesinger, A. 2013, MNRAS, 436, 1555

Frebel, A., Aoki, W., Christlieb, N., et al. 2005, Natur, 434, 871

Glover, S. C. O., \& Brand, P. W. J. L. 2001, MNRAS, 321, 385

Glover, S. C. O., \& Jappsen, A.-K. 2007, ApJ, 666, 1

Greif, T. H., Bromm, V., Clark, P. C., et al. 2012, MNRAS, 424, 399

Greif, T. H., Glover, S. C. O., Bromm, V., \& Klessen, R. S. 2009a, MNRAS, 392, 1381

Greif, T. H., Glover, S. C. O., Bromm, V., \& Klessen, R. S. 2010, ApJ, 716, 510 Greif, T. H., Jhonson, J. L., Klessen, R. S., \& Bromm, V. 2009b, MNRAS, 399,639

Greif, T. H., Springel, V., White, S., et al. 2011, ApJ, 737, 75

Haiman, Z., Thoul, A. A., \& Loeb, A. 1996, ApJ, 464, 523

Hasegawa, K., Umemura, M., \& Susa, H. 2009, MNRAS, 445

Heger, A., \& Woosley, S. E. 2002, ApJ, 567, 532

Heger, A., \& Woosley, S. E. 2010, ApJ, 724, 341

Hirano, S., Hosokawa, T., Yoshida, N., et al. 2014, ApJ, 781, 60

Hosokawa, T., Omukai, K., Yoshida, N., \& Yorke, H. W. 2011, Sci, 334, 1250

Jeon, M., Pawlik, A. H., Bromm, V., \& Milosavljević, M. 2014, MNRAS, 440, 3778

Jeon, M., Pawlik, A. H., Greif, T. H., et al. 2012, ApJ, 754, 34

Johnson, J. L., \& Bromm, V. 2007, MNRAS, 374, 1557

Johnson, J. L., Dalla Vecchia, C., \& Khochfar, S. 2013, MNRAS, 428, 1857

Johnson, J. L., Greif, T. H., Bromm, V., Klessen, R. S., \& Ippolito, J. 2009, MNRAS, 399, 37

Karlsson, T., Bromm, V., \& Bland-Hawthorn, J. 2013, RvMP, 85, 809

Karlsson, T., Johnson, J. L., \& Bromm, V. 2008, ApJ, 679, 6

Komatsu, E., Dunkley, J., Nolta, M. R., et al. 2009, ApJS, 180, 330

Kudritzki, R. P. 2002, ApJ, 577, 389

Kuhlen, M., \& Madau, P. 2005, MNRAS, 363, 1069

Langer, N. 2012, ARA\&A, 50, 107

Larson, R. B. 2003, RPPh, 66, 1651

Loeb, A., \& Furlanetto, S. 2012, The First Galaxies in the Universe (Princeton, NJ: Princeton Univ. Press)

Machacek, M. E., Bryan, G. L., \& Abel, T. 2003, MNRAS, 338, 273

Maio, U., Ciardi, B., Dolag, K., Tornatore, L., \& Khochfar, S. 2010, MNRAS, 407, 1003

Mo, H., van den Bosch, F. C., \& White, S. 2010, Galaxy Formation and Evolution (Cambridge: Cambridge Univ. Press)

Nakamura, F., \& Umemura, M. 2001, ApJ, 548, 19

Omukai, K., \& Palla, F. 2003, ApJ, 589, 677

Omukai, K., Tsuribe, T., Schneider, R., \& Ferrara, A. 2005, ApJ, 626, 627

O'Shea, B. W., Abel, T., Whalen, D., \& Norman, M. L. 2005, ApJL, 628, L5

O'Shea, B. W., McKee, C. F., Heger, A., \& Abel, T. 2008, in AIP Conf. Ser. 990, First Stars III, ed. B. W. O'Shea, \& A. Heger (Melville, NY: AIP)

Ricotti, M., Gnedin, N. Y., \& Shull, J. M. 2002, ApJ, 575, 49

Ritter, J. S., Safranek-Shrader, C., Gnat, O., Milosavljević, M., \& Bromm, V. 2012, ApJ, 761, 56

Sakuma, M., \& Susa, H. 2009, ApJ, 698, 155

Scannapieco, E., Madau, P., Woosley, S., Heger, A., \& Ferrara, A. 2005, ApJ, 633, 1031

Schaerer, D. 2002, A\&A, 382, 28

Shu, F. H., Adams, F. C., \& Lizano, S. 1987, ARA\&A, 25, 23

Shull, J. M., \& van Steenberg, M. E. 1985, ApJ, 298, 268

Springel, V. 2005, MNRAS, 364, 1105

Stacy, A., \& Bromm, V. 2013, MNRAS, 433, 1094

Stacy, A., Greif, T. H., \& Bromm, V. 2010, MNRAS, 403, 45

Stacy, A., Greif, T. H., \& Bromm, V. 2012, MNRAS, 422, 290

Susa, H. 2007, ApJ, 659, 908

Sutherland, R. S., \& Dopita, M. A. 1993, ApJS, 88, 253

Tegmark, M., Silk, J., Rees, M. J., et al. 1997, ApJ, 474, 1

Tumlinson, J. 2006, ApJ, 641, 1

Turk, M. J., Abel, T., \& O’Shea, B. 2009, Sci, 325, 601

Umeda, H., \& Nomoto, K. 2003, Natur, 422, 871

Whalen, D., Abel, T., \& Norman, M. L. 2004, ApJ, 610, 14

Whalen, D., Hueckstaedt, R. M., \& McConkie, T. O. 2010, ApJ, 712, 101 
Whalen, D. J., Smidt, J., Even, W., et al. 2014, ApJ, 781, 106

Whalen, D., van Veelen, B., O'Shea, B. W., \& Norman, M. L. 2008, ApJ, 682,49

Wiklind, T., Mobasher, B., \& Bromm, V. (ed.) 2013, Astrophysics and Space Science Library, Vol. 396 (Berlin: Springer)

Wise, J. H., \& Abel, T. 2008, ApJ, 685, 40

Wise, J. H., \& Cen, R. 2009, ApJ, 693, 984
Wise, J. H., Demchenko, V. G., Halicek, M. T., et al. 2014, MNRAS, 442, 2560

Wise, J. H., Turk, M. J., Norman, M. L., \& Abel, T. 2012, ApJ, 745, 50

Woosley, S. E. 1993, ApJ, 405, 273

Xu, H., Ahn, K., Wise, J. H., Norman, M. L., \& O’Shea, B. W. 2014, ApJ, 791,110

Xu, H., Wise, J. H., \& Norman, M. L. 2013, ApJ, 773, 83

Yoshida, N., Oh, S. P., Kitayama, T., \& Hernquist, L. 2007, ApJ, 663, 687 